\title{
Understanding data noise in gravity field recovery on the basis of inter-satellite ranging measurements acquired by the satellite gravimetry mission GRACE
}

\author{
Pavel Ditmar • João Teixeira da Encarnação • \\ Hassan Hashemi Farahani
}

Received: 24 March 2011 / Accepted: 9 November 2011 / Published online: 26 November 2011

(C) The Author(s) 2011. This article is published with open access at Springerlink.com

\begin{abstract}
Spectral analysis of data noise is performed in the context of gravity field recovery from inter-satellite ranging measurements acquired by the satellite gravimetry mission GRACE. The motivation of the study is two-fold: (i) to promote a further improvement of GRACE data processing techniques and (ii) to assist designing GRACE follow-on missions. The analyzed noise realizations are produced as the difference between the actual GRACE inter-satellite range measurements and the predictions based on state-of-the-art force models. The exploited functional model is based on the so-called "range combinations," which can be understood as a finite-difference analog of inter-satellite accelerations projected onto the line-of-sight connecting the satellites. It is shown that low-frequency noise is caused by limited accuracy of the computed GRACE orbits. In the first instance, it leads to an inaccurate estimation of the radial component of the inter-satellite velocities. A large impact of this component stems from the fact that it is directly related to centrifugal accelerations, which have to be taken into account when the measured range-accelerations are linked with inter-satellite accelerations. Another effect of orbit inaccuracies is a miscalculation of forces acting on the satellites (particularly, the one described by the zero-degree term of the Earth's gravitational field). The major contributors to the noise budget at high frequencies (above $9 \mathrm{mHz}$ ) are (i) ranging sensor errors and (ii) limited knowledge of the Earth's static gravity field at high degrees. Importantly, we show that updating the model
\end{abstract}

P. Ditmar $(\bowtie) \cdot$ J. Teixeira da Encarnação $\cdot$ H. Hashemi Farahani Delft University of Technology, Kluyverweg 1,

2629 HS, Delft, The Netherlands

e-mail: P.G.Ditmar@tudelft.nl

J. Teixeira da Encarnação

e-mail: J.G.deTeixeiradaEncarnacao@tudelft.nl

H. Hashemi Farahani

e-mail: H.Hashemi@tudelft.nl of the static field on the basis of the available data must be performed with a caution as the result may not be physical due to a non-unique recovery of high-degree coefficients. The source of noise in the range of intermediate frequencies $(1-9 \mathrm{mHz})$, which is particularly critical for an accurate gravity field recovery, is not fully understood yet. We show, however, that it cannot be explained by inaccuracies in background models of time-varying gravity field. It is stressed that most of the obtained results can be treated as sufficiently general (i.e., applicable in the context of a statistically optimal estimation based on any functional model).

Keywords Satellite-to-satellite tracking $\cdot \mathrm{K}$-band ranging . Temporal gravity field variations $\cdot$ Mass transport . Earth's system · GRACE follow-on

\section{Introduction}

Gravity Recovery and Climate Experiment (GRACE) satellite mission (Tapley et al. 2004) was launched in 2002 to globally collect observations of the Earth's gravity field with unprecedented accuracy and spatial resolution. The mission consists of two twin satellites separated by a $200-\mathrm{km}$ offset and following each other in a nearly polar orbit at the altitude of about $500 \mathrm{~km}$. There are two primary mission objectives: (i) to improve static models of the Earth's gravity field and geoid; and (ii) to monitor global mass transport in the Earth's system by observing the associated temporal gravity field variations.

On the basis of GRACE data, a whole series of static gravity field models was produced, including GGM02 (Tapley et al. 2005), EIGEN-GL04C (Förste et al. 2008), and EGM2008 (Pavlis et al. 2008, frequently shortened to EGM08), which demonstrated a dramatic improvement 
compared to the pre-GRACE era. Furthermore, models of temporal gravity field variations are routinely produced from GRACE data (typically, with the temporal resolution of one month) (Bettadpur 2007; Flechtner et al. 2010; Ilk et al. 2005; Luthcke et al. 2006; Lemoine et al. 2007; Liu et al. 2010). Such models are used, in particular, for monitoring the redistribution of water in the Earth's system. This information is critical for better understanding key climate change indicators, such as ice-sheet mass balance, terrestrial water-storage change, sea-level rise, and ocean circulation.

Technically, the observation principle of most of satellite gravimetry missions, including GRACE, is based on the Newton's second law: $\mathbf{a}=\mathbf{f}$, where $\mathbf{a}$ is the observed satellite acceleration, and $\mathbf{f}$ is the specific force (i.e., the force divided by the satellite mass) acting on the satellite. The satellite accelerations are derived (explicitly or implicitly) from the observed satellite motion. To track the satellite motion as precisely as possible, the GRACE satellites are equipped with the K-Band Ranging (KBR) system, which continuously measures variations of the inter-satellite distance with a micrometer-level accuracy. The forces exerted onto the satellites have both gravitational and non-gravitational origin. The latter ones are measured with on-board accelerometers, so that their influence can be corrected for in the course of data processing. The remaining signals reflect not only the static gravitational field of the Earth, but also all the mass transport processes, including high-frequency ones (i.e. taking place at a time scale of days or even hours). Such high-frequency signals play a role of an additional source of noise, which may cause unpredictable and large distortions of the produced gravity field models; such an effect is called "temporal aliasing". To minimize temporal aliasing, the satellite data are cleaned from high-frequency signals at the pre-processing stage, for which purpose appropriate background models are used. The list of such models includes a model of ocean tides, e.g. FES2004 (Lyard et al. 2006) and a model of non-tidal mass re-distribution in the atmosphere and ocean, such as the so-called Atmosphere and Ocean De-aliasing (AOD1B) product (Flechtner 2007).

In spite of remarkable achievements, the GRACE mission is not free from certain limitations. In particular, this concerns the accuracy of the produced gravity field and mass change models. In the absence of regularization or low-pass filtering, noise in produced models increases with spherical harmonic degree (i.e., as the spatial scale diminishes). For a typical monthly model based on GRACE data, noise reaches the level of $1 \mathrm{~mm}$ at degree 60 and $10 \mathrm{~mm}$ at degree 100 in terms of RMS geoid height error per degree (Liu 2008, pp. 175-176). Furthermore, errors in the obtained models change within a given degree by orders of magnitude, depending on spherical harmonic order. Sectorial and nearly sectorial coefficients in the models are estimated with particularly large uncertainties. In the spatial domain, these errors form a pronounced along-track stripes resembling a tiger skin. One of the major causes of this effect is an anisotropic sensitivity of the GRACE mission. The observation of inter-satellite range variations delivers, in essence, information about the differences of the Earth's gravitation at the satellite locations. This difference can be interpreted as an approximation of the along-track component of the gravitation field gradient. Since the GRACE orbits are nearly polar, the along-track direction is most of the time almost parallel to the direction North-South. However, a function on a sphere, generally speaking, cannot be uniquely restored from only the North-South component of its gradient. Any function that depends only on the longitude can be added to the actual one without changing the values of the North-South gradient component. Fortunately, such a reasoning is based on a certain idealization; the actual situation is not that dramatic. In particular, the Earth's rotation always results in the presence of an East-West component in the inter-satellite offset vector. Nevertheless, sensitivity of the GRACE observations remains rather anisotropic: East-West variations of the gravitational field are sensed much worse than North-South ones. The anisotropic sensitivity is a factor that amplifies the influence of noise in GRACE inter-satellite range measurements.

Unfortunately, the information contents and error budget of the GRACE data are not fully understood yet. Naturally, one of the contributors to the noise budget is sensor noise, particularly the one associated with the KBR system and the on-board accelerometers. A number of authors have estimated how noise of this type propagates into monthly gravity field solutions and demonstrated that its influence is modest. For instance, according to $\operatorname{Kim}(2000$, p. 238) and Han et al. (2004), the corresponding geoid height error per degree reaches $0.1 \mathrm{~mm}$ at degree 60 . Moreover, Thompson et al. (2004) and Ray and Luthcke (2006) have demonstrated an even lower level (about $0.03 \mathrm{~mm}$ at degree 60 in terms of geoid height error per degree). Prior to the launch of the GRACE mission, the expected level of total noise in the monthly solutions was close to the sensor-only based estimations mentioned above (the so-called, "GRACE baseline") (Kim 2000). This is because the sensor noise was considered as the dominant factor limiting the accuracy of the monthly models to be obtained. Later it became clear, however, that temporal aliasing errors may either be comparable with the impact of sensor noise or even exceed it several times (depending on the assumptions about noise in the background models and about sensor noise) (Thompson et al. 2004; Han et al. 2004; Ray and Luthcke 2006). At the same time, the analysis presented in the literature suggests that noise in background models cannot explain the actually observed noise in GRACE data: the latter one is a few times or even by an order of magnitude higher.

The major objective of this study is to carry out an in-depth analysis of noise in GRACE data and, ideally, identify other 
noise sources than sensors and inaccuracies in background models. The motivation of such a research is twofold. First, it may result in a further improvement of GRACE data processing techniques. Second, it might be helpful in designing GRACE follow-on missions. Investigations of the optimal design of such a mission started a few years ago (Sneeuw et al. 2008; Pierce et al. 2008; van Dam et al. 2008; Wiese et al. 2009) and have become, by this moment, the focus of research efforts of numerous groups worldwide. The key element of such researches is assessing the performance of various mission scenarios. However, this is only possible if all the major contributors to the noise budget are well understood and can be reliably reproduced in the course of simulations. The GRACE mission can be considered as a unique source of such knowledge.

In order to reach the study goals, we analyze noise in GRACE Level-1B data (Case et al. 2004) in the spectral domain. In different frequency bands, we compare properties of the actually observed noise level with those of a synthetic noise of a various origin. In this way, most probable sources of noise in GRACE data can be identified.

The outline of the paper is as follows: in Sect. 2, we present the theoretical basis of the conducted analysis. We give a precise definition of signal and noise, explain the exploited functional model, and present the generated noise realizations used in the study. In Sect. 3 we discuss the contribution of inaccuracies in the computed GRACE orbits to the noise budget. Other observed and potential contributors to the noise budget are analyzed in Sect. 4. Finally, Sect. 5 is left for discussion and conclusions.

\section{Production of noise realizations in GRACE data}

The major focus of this section is preparation of realizations of noise in GRACE data. In Sect. 2.1 we introduce the concept of data noise in a very general sense. Sect. 2.2 is devoted to the issue of noise in the context of GRACE KBR data. We explain the adopted functional model, which defines the "data" in the context of the presented study. Besides, we present in detail our procedure for production of noise realizations, which are used in the rest of the paper. Furthermore, we discuss the potential contributors in the noise budget. In particular, we present an explicit analytic link between errors in background force models and noise in the data. Finally, Sect. 2.3 is devoted to more practical aspects. We present the time interval considered in our study and make a preliminary analysis of the obtained noise realizations in the spectral domain.

\subsection{What is noise?}

In general, the goal of data processing is to compute parameters of a model describing the phenomenon under consider- ation. In an ideal world, the collected observations $\mathbf{0}$ are fully consistent with the forecasts $\mathbf{f}$ based on a properly specified set of model parameters:

$\mathbf{o}=\mathbf{f}$.

In reality, the actually acquired observations $\hat{\mathbf{o}}$ contain noise $\delta_{\boldsymbol{O}}$ due to, e.g., sensor imperfectness:

$\hat{\mathbf{o}}=\mathbf{o}+\boldsymbol{\delta}_{\boldsymbol{O}}$.

Furthermore, the actual forecasts $\hat{\mathbf{f}}$ are also contaminated by noise $\delta_{\boldsymbol{F}}$ due to, e.g., an inadequate model parameterization or inaccurate knowledge of sensor positions and attitude:

$\hat{\mathbf{f}}=\mathbf{f}+\delta_{F}$.

Then, the total data noise $\delta$ can be defined as the difference between the actual observations and the actual forecasts:

$\delta=\hat{\mathbf{o}}-\hat{\mathbf{f}}=\delta_{O}-\delta_{F}$.

In the context of GRACE data, such a general definition of data noise allows all the contributors to the noise budget to be taken into account. In particular, the term $\boldsymbol{\delta}_{\boldsymbol{F}}$ accounts for residual rapid gravity field changes left in the data due to inaccuracies in the background models. In addition, this term also contains noise caused by an inaccurate computation of satellite orbits which are needed to compute the forecasts $\hat{\mathbf{f}}$. We emphasize that we prefer to consider errors in the satellite orbits separately from the errors in background models, even though the latter are definitely one of the causes of the former errors. A further discussion of this issue, as well as a more strict separation of the noise budget into individual contributors, can be found in the sections below.

\subsection{Methodology}

In conducting this study, we have used the experience collected in the course of producing the Delft Mass Transport model DMT-1 (Liu et al. 2010). That model is composed of a set of monthly solutions, each of which consists of spherical harmonic coefficients spanning the degree range from 2 to 120. Each solution has been subject to the post-processing with a statistically optimal Wiener-type filter based on full covariance matrices of signal and noise (Klees et al. 2008; Liu et al. 2010).

\subsubsection{Force model and computation of GRACE orbits}

It order to compute forecasted data, it is necessary, in particular, to introduce an "ideal" force model (i.e. a model that represents state-of-the-art knowledge about the forces which may influence the satellite motion). The force model exploited in the current study is similar to that used in the production of the DMT-1 model. It includes the EIGENGL04C model (Förste et al. 2008) of the static gravity field 
of the Earth; commonly-used models of N-body perturbations and tides, including the FES2004 model of ocean tides (Lyard et al. 2006); the AOD1B product (Flechtner 2007) describing non-tidal mass re-distribution in the atmosphere and oceans; a model of relativistic effects defined according to IERS 2003 conventions (McCarthy and Petit 2004), as well as non-gravitational acceleration observations collected by the on-board GRACE accelerometers. Furthermore, the DMT-1 model itself is added to the exploited force model as a source of information about mass re-distribution at a monthly and longer time scales. It is worth noticing that linear trends in low-degree coefficients that are a part of the EIGEN-GL04C model are not included because those trends are already contained in the DMT-1 solutions (Liu et al. 2010).

The composed force model is used to produce GRACE dynamic orbits by fitting them to high-precision kinematic orbits computed by Dr. Qile Zhao. The dynamic orbits are computed in the form of 6-hour arcs. In the course of the orbit computation, 12 parameters per arc are estimated: the initial state vector (6 parameters); the bias in accelerometer data per component ( 3 parameters); and the scaling factor applied to accelerometer data per component ( 3 parameters). Positioning And Navigation Data Analyst (PANDA) software (Zhao 2004) has been used to compute the dynamic orbits. A similar approach was used earlier for the production of the DMT-1 model, including the kinematic orbits used as an input, the numerical procedure, and the software.

\subsubsection{Terminology and notation}

Now, let us discuss the terminology to be used in the rest of Sect. 2 and further on. First of all, let us introduce a local frame at a given observation epoch such that (i) the $\mathrm{x}$-axis is parallel to the Line-Of-Sight (LOS), which is directed from the trailing satellite to the leading one; (ii) the $\mathrm{z}$-axis is directed upwards, being orthogonal to the $\mathrm{x}$-axis and belonging to the plane defined by the satellites' locations and the center of the Earth; and (iii) the y-axis is orthogonal to $\mathrm{x}$ - and $\mathrm{z}$-axes, complementing the frame to a right-handed one. The origin of the frame coincides with the center of the Earth.

We assume that all the quantities under consideration are given with a constant sampling interval $\Delta t$. A quantity with the lower index $i$ corresponds to the observation time $t_{i}:=$ $t_{0}+i \Delta t$, where $t_{0}$ is an initial epoch $(i=1, \ldots N$ with $N$ being the total number of data). Sometimes, however, the lower index $i$ is omitted to simplify the notation.

A particular component of a vector is denoted with the corresponding upper index: $(x),(y)$, or $(z)$. In certain instances, the local frame defined for a time $t_{i}$ is used to introduce an instantaneous inertial frame, which is exploited to identify vector components a few moments before and after time $t_{i}$. In those cases, the upper index is supplied with the subscript $i$.

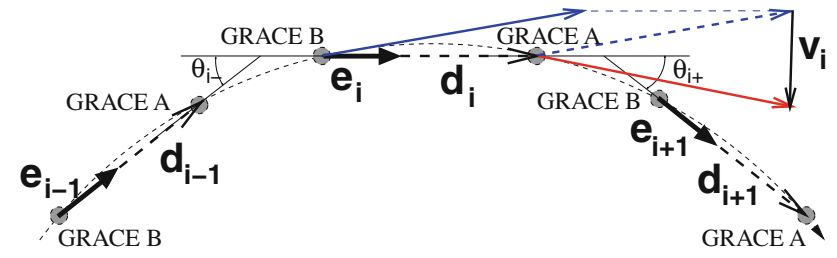

Fig. 1 Basic parameters utilized in the adopted functional model: Direction of line-of-sight vectors at three successive epochs $\left(\mathbf{e}_{\mathbf{i}-\mathbf{1}}, \mathbf{e}_{\mathbf{i}}, \mathbf{e}_{\mathbf{i}+\mathbf{1}}\right)$; inter-satellite offset vectors at three successive epochs $\left(\mathbf{d}_{\mathbf{i}-\mathbf{1}}, \mathbf{d}_{\mathbf{i}}, \mathbf{d}_{\mathbf{i}+\mathbf{1}}\right)$; inter-satellite velocity vector at the epoch $i\left(\mathbf{v}_{\mathbf{i}}\right)$; and the angles between the successive line-of-sight vectors $\left(\theta_{i-}\right.$ and $\left.\theta_{i+}\right)$ used for computing the range combinations (Eq. 5). The red and blue arrows denote the velocity vectors of the leading and trailing satellite, respectively

The inter-satellite offset vector is denoted as $\mathbf{d}$. It is directed from the trailing satellite to the leading one (Fig. 1). In other words, the vector $\mathbf{d}$ is approximately parallel to the velocity vectors of the individual satellites. The absolute value of the vector $\mathbf{d}$, which is called "inter-satellite range", is denoted as $\rho$. The unit vector defining the LOS direction is denoted as $\mathbf{e}: \mathbf{e}:=\frac{\mathbf{d}}{\rho}$. The inter-satellite velocity vector (v) and the inter-satellite acceleration vector (a) are defined as the vector difference between, respectively, the velocity and the acceleration of the leading and the trailing satellite: $\mathbf{v}:=\dot{\mathbf{d}} ; \mathbf{a}:=\ddot{\mathbf{d}}$. Further terms and symbols will be introduced as needed.

\subsubsection{Functional model}

The exploited functional model is also similar to that used for the production of the DMT-1 model. It is based on the so-called range combinations, each of which is formed as a linear combination of three successive range measurements $\rho_{i-1}, \rho_{i}$, and $\rho_{i+1}$ :

$\bar{a}_{i}^{(x)}=\frac{\cos \theta_{i-} \cdot \rho_{i-1}-2 \rho_{i}+\cos \theta_{i+} \cdot \rho_{i+1}}{(\Delta t)^{2}}$,

where $\theta_{i-}$ and $\theta_{i+}$ are the angles between the LOS at epoch $i$, and the LOSs at the epochs $i-1$ and $i+1$, respectively (see Fig. 1).

It is worth mentioning that GRACE K-band range data contain an unknown bias. In order to apply Eq. (5) in practice, we produce the de-biased ranges at an initial stage of GRACE data processing, as explained by Liu et al. (2010).

The resulting quantity of Eq. (5), $\bar{a}_{i}^{(x)}$, can be interpreted as a weighted average of the inter-satellite accelerations in the time interval $\left[t_{i}-\Delta t ; t_{i}+\Delta t\right]$ projected onto the LOS at the epoch $i$. Conceptually, the average accelerations $\bar{a}_{i}^{(x)}$ can also be produced from a time series of point-wise intersatellite accelerations by applying an appropriate filter, called by Ditmar and van Eck van der Sluijs (2004) an "averaging filter". Let $\mathbf{a}^{\left(\mathbf{x}_{\mathbf{i}}\right)}$ be a vector composed of $2 n+1$ point-wise 
inter-satellite accelerations projected on the axis $x_{i}$ in the inertial frame (this axis is defined such that it coincides with the $\mathrm{x}$-axis in the local frame at the time $t_{i}$ ). The time interval covered by the considered point-wise inter-satellite accelerations is defined as $\left[t_{i}-n \Delta t ; t_{i}+n \Delta t\right]$. Then, the average inter-satellite acceleration $\bar{a}_{i}^{(x)}$ can be computed as

$\bar{a}_{i}^{(x)}=\mathbf{E}^{T} \mathbf{a}^{\left(\mathbf{x}_{\mathbf{i}}\right)}$,

where the vector $\mathbf{E}$ of the length $2 n+1$ is composed of constant (pre-defined) averaging filter coefficients, the number $2 n$ being called the order of the averaging filter. For instance, for the second-order averaging filter, it holds: $\mathbf{E}=$ $(1 / 12 ; 5 / 6 ; 1 / 12)^{T}$. In other words, a time series of average inter-satellite accelerations can be interpreted as a slightly smoothed time series of point-wise inter-satellite accelerations. At low frequencies, average and point-wise accelerations are almost identical.

It is worth adding that the concept of range combinations is very similar to the concept of 3-D average accelerations of a single satellite (Ditmar and van Eck van der Sluijs 2004), which has been successfully applied, for instance, to gravity field modeling on the basis of CHAllenging Minisatellite Payload (CHAMP) satellite data (Ditmar et al. 2006).

We find the functional model based on range combination perfectly suited for an analysis of errors in the data. This is because errors in force models directly propagate into the data at the vicinity of the locations where the force model is inaccurate. In other words, the exploited functional model is "local". This is in contrast to ranges or range-rates, which depend on the forces not only at the vicinity of the current point, but also at the points visited by the satellites in the past. Usage of a local functional model might help in relating the observed data errors to the force models that are responsible for those errors.

In addition, the use of range combinations allows us to extensively exploit the algorithms designed earlier for the production of the DMT-1 model. It is worth mentioning that gravity field solutions produced from range combinations demonstrate a similar accuracy as the solutions obtained with other techniques (Liu 2008, pp. 175-176).

\subsubsection{Link with the "classical" acceleration approach}

There is also a close link between the approach based on range combinations and the "classical" acceleration approach (Rummel 1979). According to the latter approach, the alongtrack component of the point-wise inter-satellite accelerations can be derived from GRACE measurements as follows:

$a_{i}^{(x)}=\ddot{\rho}_{i}-\frac{1}{\rho_{i}}\left(v_{i}^{(y)}+v_{i}^{(z)}\right)^{2}$.

The first term in the right-hand side of this expression is the range-acceleration (the second time-derivative of the range), which is directly derived from the KBR data. The second term represents the centrifugal accelerations, which are the consequence of the fact that the local frame, which is coupled with LOS direction, is non-inertial. The centrifugal accelerations have to be derived from estimated satellite orbits. By neglecting the out-of-plane motion, we can represent the second term in Eq. (7) as

$a_{i}^{(\mathrm{cent}, x)}=-\frac{1}{\rho_{i}}\left(v_{i}^{(y)}+v_{i}^{(z)}\right)^{2} \approx-\frac{1}{\rho_{i}}\left(v_{i}^{(z)}\right)^{2}$.

Now, let us return to the concept of range combinations and re-write Eq. (5) as follows:

$$
\begin{aligned}
\bar{a}_{i}^{(x)}= & \frac{\rho_{i-1}-2 \rho_{i}+\rho_{i+1}}{(\Delta t)^{2}} \\
& -\frac{\left(1-\cos \theta_{i-}\right) \cdot \rho_{i-1}+\left(1-\cos \theta_{i+}\right) \cdot \rho_{i+1}}{(\Delta t)^{2}} .
\end{aligned}
$$

Obviously, the first term in this expression is a finite-difference analog of the range-acceleration, the first term in the left-hand side of Eq. (7). Let us consider the second term. Taking into account the identity $1-\cos \theta=2 \sin ^{2}\left(\frac{\theta}{2}\right)$, we can re-write it as

$$
-\frac{2 \sin ^{2}\left(\frac{\theta_{i-}}{2}\right) \cdot \rho_{i-1}+2 \sin ^{2}\left(\frac{\theta_{i+}}{2}\right) \cdot \rho_{i+1}}{(\Delta t)^{2}} .
$$

Let us introduce the angle $\theta(t)$ as the angle between the LOS direction at an arbitrary moment and the LOS direction at the time $t_{i}\left(\theta(t)<0\right.$ for $t<t_{i} ; \theta(t)>0$ for $\left.t>t_{i}\right)$. Variations of the angle $\theta(t)$ mostly reflect the rotation of the satellites in a given orbital plane. Therefore, it follows from simple geometrical considerations that

$\sin \theta(t) \approx-\frac{d^{\left(z_{i}\right)}(t)}{\rho(t)}$,

where $d^{\left(z_{i}\right)}(t)$ is the $\mathrm{z}$-component of the current inter-satellite offset vector (the $\mathrm{z}$-axis being defined in concert with the local frame at the time $t_{i}$ ). Let us further assume that the function $\theta(t)$ is approximately linear in the time interval $\left[t_{i}-\right.$ $\left.\Delta t ; t_{i}+\Delta t\right]$. In this case, the angles $\frac{\theta_{i-}}{2}$ and $\frac{\theta_{i+}}{2}$ reflect the LOS orientation at the times $t_{i}-\Delta t / 2$ and $t_{i}+\Delta t / 2$, respectively, i.e.,

$$
\left\{\begin{array}{l}
\sin \left(\frac{\theta_{i-}}{2}\right) \approx-\frac{d^{\left(z_{i}\right)}\left(t_{i}-\Delta t / 2\right)}{\rho\left(t_{i}-\Delta t / 2\right)} \\
\sin \left(\frac{\theta_{i+}}{2}\right) \approx-\frac{d^{\left(z_{i}\right)}\left(t_{i}+\Delta t / 2\right)}{\rho\left(t_{i}+\Delta t / 2\right)} .
\end{array}\right.
$$

In addition, let us assume that $d^{\left(z_{i}\right)}(t)$ is also a linear function at the vicinity of the time $t_{i}$. Since by definition $d^{\left(z_{i}\right)}\left(t_{i}\right)=0$, we readily obtain 
Table 1 Procedures for computing the observed range combinations, forecasted range combinations, and actual noise realizations

\begin{tabular}{|c|c|c|}
\hline Product & Input & Procedure \\
\hline $\begin{array}{c}\text { Observed range } \\
\text { combinations }\end{array}$ & $\begin{array}{l}\text { GRACE KBR data } \\
\text { LOS directions based } \\
\text { on the dynamic orbits }\end{array}$ & Double numerical differentiation, Eq. (5) \\
\hline $\begin{array}{l}\text { Forecasted range } \\
\text { combinations } \\
\text { (option 1) }\end{array}$ & $\begin{array}{l}\text { Inter-satellite ranges and } \\
\text { LOS directions based } \\
\text { on the dynamic orbits }\end{array}$ & Double numerical differentiation, Eq. (5) \\
\hline $\begin{array}{l}\text { Forecasted range } \\
\text { combinations } \\
\text { (option 2) }\end{array}$ & $\begin{array}{l}\text { "Ideal" force model } \\
\text { Dynamic orbits }\end{array}$ & $\begin{array}{l}\text { Computation of point-wise } \\
\text { inter-satellite accelerations } \\
\text { Application of the averaging filter (cf. Eq. 6) } \\
\text { Projecting onto the LOS }\end{array}$ \\
\hline Actual noise realizations & $\begin{array}{l}\text { Observed range combinations } \\
\text { Forecasted range combinations }\end{array}$ & $\begin{array}{l}\text { Subtraction of the forecasted range } \\
\text { combinations from the observed ones }\end{array}$ \\
\hline
\end{tabular}

$$
\left\{\begin{aligned}
d^{\left(z_{i}\right)}\left(t_{i}-\Delta t / 2\right) & \approx-\frac{v_{i}^{(z)} \Delta t}{2} \\
d^{\left(z_{i}\right)}\left(t_{i}+\Delta t / 2\right) & \approx \frac{v_{i}^{(z)} \Delta t}{2},
\end{aligned}\right.
$$

where $v_{i}^{(z)}$ is the z-component of the inter-satellite velocity vector in the local frame at the time $t_{i}$. Then, the substitution of Eqs. (12) and (13) into (10) allows us to re-write the second term in Eq. (9) as follows:

$$
-\frac{\left(v_{i}^{(z)}\right)^{2}}{2}\left(\frac{\rho_{i-1}}{\rho^{2}\left(t_{i}-\Delta t / 2\right)}+\frac{\rho_{i+1}}{\rho^{2}\left(t_{i}+\Delta t / 2\right)}\right) .
$$

Assuming that the inter-satellite range shows only minor variations (i.e. that $\rho\left(t_{i}-\Delta t\right) \approx \rho\left(t_{i}-\Delta t / 2\right) \approx \rho\left(t_{i}\right) \approx$ $\left.\rho\left(t_{i}+\Delta t / 2\right) \approx \rho\left(t_{i}+\Delta t\right)\right)$, we finally arrive at $-\frac{1}{\rho_{i}}\left(v_{i}^{(z)}\right)^{2}$. This is equal to the centrifugal acceleration term given by Eq. (8). We recall that we arrived at this result having made the assumptions that the angle $\theta$ and the z-component of the inter-satellite offset vector change linearly, whereas the intersatellite range is constant. Obviously, all these assumptions are satisfied in a sufficiently close vicinity of the time $t_{i}$. This means that range combinations can be considered as a finitedifference approximation of the along-track component of inter-satellite accelerations, Eq. (7). The data of these two types become equivalent as $\Delta t \rightarrow 0$.

\subsubsection{Computation of data noise}

In the conducted study, realizations of GRACE data noise are obtained by computing the difference between the observed and forecasted range combinations. The observed range combinations are computed directly with Eq. (5) on the basis of real GRACE KBR data, which are provided with 5-s sam- pling (the Nyquist frequency is $100 \mathrm{mHz}$ ). These data are supplied with information about the LOS directions, which is derived from the produced dynamic orbits. As far as the forecasted range combinations are concerned, two approaches can be applied. The first one is to reuse for that purpose Eq. (5), where the observed ranges are replaced with those derived from the dynamic orbits. The second approach is to compute 3-D point-wise accelerations along the computed orbits, and then produce on their basis average inter-satellite accelerations, making use of the averaging filter, Eq. (6). According to Ditmar and van Eck van der Sluijs (2004), these two approaches are equivalent. We have implemented both approaches and verified that the obtained results indeed coincide (within a computation error). In the analytical analysis of forecast errors, which is presented in further sections, the second approach is exploited as more straightforward. A summary of the exploited algorithms for producing the observed range combinations, forecasted range combinations, and noise realizations is given in Table 1.

Noise in range combinations can occur due to several factors. One of them is ranging sensor errors. Another one is errors in the orbits, which result in an inaccurate definition of the positions at which the force model is estimated. In addition, noise of this type leads to an inaccurate estimation of the LOS directions described by the angles $\theta_{i-}$ and $\theta_{i+}$. One more factor is inaccuracies in the exploited "ideal" force model itself. A more systematic inventory of potential sources of noise in range combinations is presented in the next subsection.

\subsubsection{Inventory of potential sources of noise in range combinations}

As it is already explained in Sect. 2.1, the total data noise $\delta$ is composed of the observation noise $\delta_{\mathrm{O}}$ and the forecast 
noise $\delta_{\mathrm{F}}$ (the index $i$ is omitted for simplicity). First of all, let us consider the observation noise. As it is explained in Sect. 2.2.4, an observation, i.e., a range combination, can be represented as the finite-difference analog of range-acceleration and a correction for centrifugal acceleration. Consequently, we split the observation noise into the ranging noise $\delta_{\mathrm{R}}$ and the correction noise $\delta_{\mathrm{C}}$ :

$\delta_{\mathrm{O}}=\delta_{\mathrm{R}}+\delta_{\mathrm{C}}$.

According to Eq. (9), the ranging noise at the time $t_{i}$ depends only on the inaccuracies $\delta \rho_{i-1}, \delta \rho_{i}$, and $\delta \rho_{i+1}$ in the measured ranges:

$\delta_{\mathrm{R}}=\frac{\delta \rho_{i-1}-2 \delta \rho_{i}+\delta \rho_{i+1}}{(\Delta t)^{2}}$.

These inaccuracies mostly reflect an imperfectness of the KBR sensor. As far as the correction noise is concerned, it can be interpreted as inaccuracies in the estimated centrifugal accelerations given by Eq. (8). This means that the correction noise at time $t_{i}$ can be represented as

$\delta_{\mathrm{C}}=-\frac{2 v^{(z)}}{\rho} \delta v^{(z)}+\frac{\left(v^{(z)}\right)^{2}}{\rho^{2}} \delta \rho$.

Thus, the correction noise depends both on errors in the estimated inter-satellite velocities $v^{(z)}$ (the first term in the expression above) and on KBR sensor errors (the second term).

It is worth adding that Eq. (17), strictly speaking, must be exploited in the context of range combinations with some caution. It is based on the expression for centrifugal accelerations given by Eq. (8), which is a continuous analog of Eq. (10). This means that Eq. (17) can be directly applied to range combinations only in the range of low frequencies. In other words, all the functions and the errors involved must show only slow variations in time. In Sect. 2.3 it is shown, however, that in practice this difference may play a noticeable role only if the frequencies under consideration are comparable with the Nyquist one.

As far as the forecast noise $\delta_{\mathrm{F}}$ is concerned, it can be understood as noise in the computed force model propagated into the data. We further split this noise into the following components: (i) noise $\bar{\delta}_{\mathrm{GS}}$ caused by errors in the static gravity field model; (ii) noise $\bar{\delta}_{\mathrm{GV}}$ caused by errors in time-varying gravity field models; (iii) noise $\bar{\delta}_{\mathrm{NG}}$ in non-gravitational accelerations (predominantly caused by an imperfectness of the on-board accelerometers); (iv) the satellite position noise $\bar{\delta}_{\mathrm{P}}$; and (v) LOS orientation noise $\bar{\delta}_{\mathrm{L}}$ caused by errors in the LOS orientations. In other words

$\delta_{\mathrm{F}}=\bar{\delta}_{\mathrm{GS}}+\bar{\delta}_{\mathrm{GV}}+\bar{\delta}_{\mathrm{NG}}+\bar{\delta}_{\mathrm{P}}+\bar{\delta}_{\mathrm{L}}$.

As a link to the discussions in Sections 3 and 4, we would like to add that noise of all these types can be produced from pointwise estimates of errors in the corresponding force models by applying the averaging filter:

$$
\left\{\begin{array}{l}
\bar{\delta}_{\mathrm{GS}}=\mathbf{E}^{T} \boldsymbol{\delta}_{\boldsymbol{G S}} \\
\bar{\delta}_{\mathrm{GV}}=\mathbf{E}^{T} \boldsymbol{\delta}_{\boldsymbol{G}} \boldsymbol{} \\
\bar{\delta}_{\mathrm{NG}}=\mathbf{E}^{T} \boldsymbol{\delta}_{\boldsymbol{N}} \boldsymbol{G} \\
\bar{\delta}_{\mathrm{P}}=\mathbf{E}^{T} \boldsymbol{\delta}_{\boldsymbol{P}} \\
\bar{\delta}_{\mathrm{L}}=\mathbf{E}^{T} \boldsymbol{\delta}_{\boldsymbol{L}} .
\end{array}\right.
$$

Note that the vectors $\delta_{G S}, \delta_{G V}, \delta_{N G}, \delta_{P}$, and $\delta_{L}$ are of the length consistent with that of the averaging filter vector $\mathbf{E}$.

Let us derive more explicit expressions for the vectors $\delta_{P}=\delta_{P_{i}}$ and $\delta_{L}=\delta_{L_{i}}$. Let us denote the $p$-th element of the vector $\delta_{P_{i}}$ as $\delta_{P_{i}}^{p}(p=-n, \ldots n)$. This element describes the impact of the positioning errors at the time $t_{i+p}$. It can be further represented as

$\delta_{P_{i}}^{p}=\delta_{P_{1, i}}^{p}-\delta_{P_{2, i}}^{p}$,

where $\delta_{P_{1, i}}^{p}$ and $\delta_{P_{2, i}}^{p}$ is noise associated with the leading and trailing satellite, respectively. For both satellites, it holds:

$$
\begin{aligned}
\delta_{P_{k, i}}^{p}= & g^{\left(x_{i}\right)}\left(\mathbf{r}_{k}+\delta \mathbf{r}_{k}\right)-g^{\left(x_{i}\right)}\left(\mathbf{r}_{k}\right) \approx\left(\mathbf{G}^{\left(\mathbf{x}_{\mathbf{i}}\right)}\left(\mathbf{r}_{k}\right) \cdot \delta \mathbf{r}_{k}\right) \\
& (k=1,2),
\end{aligned}
$$

where $\mathbf{r}_{k}=\mathbf{r}_{k}\left(t_{i+p}\right)$ is the position of the $k$-th satellite at the time $t_{i+p}$ and $\delta \mathbf{r}_{k}=\delta \mathbf{r}_{k}\left(t_{i+p}\right)$ is the error in the estimate of this position. Furthermore, $g^{\left(x_{i}\right)}$ is the X-component of the total gravitational field in the inertial frame that coincides with the local frame at the time $t_{i}$. Finally, $\mathbf{G}^{\left(\mathbf{x}_{\mathbf{i}}\right)}\left(\mathbf{r}_{k}\right)$ is the vector composed of 3 elements of the gravity gradient matrix $\mathbf{G}$ (matrix of the second-order derivatives of the gravitational potential) in the aforementioned inertial frame: $\mathbf{G}^{\left(\mathbf{x}_{\mathbf{i}}\right)}=\left(G^{\left(x_{i}, x_{i}\right)}, G^{\left(x_{i}, y_{i}\right)}, G^{\left(x_{i}, z_{i}\right)}\right)^{T}$.

Similarly, for LOS orientation noise it holds:

$\delta_{L_{i}}^{p}=\delta_{L_{1, i}}^{p}-\delta_{L_{2, i}}^{p}$.

where $\delta_{L_{1, i}}^{p}$ and $\delta_{L_{2, i}}^{p}$ is noise associated with the leading and trailing satellite, respectively. In both cases, it holds:

$\delta_{L_{k, i}}^{p}=\left(\mathbf{g}\left(\mathbf{r}_{\mathbf{k}}\right) \cdot \boldsymbol{\delta} \mathbf{e}\right) \quad(k=1,2)$,

where $\mathbf{g}\left(\mathbf{r}_{k}\right)=\mathbf{g}\left(\mathbf{r}_{k}\left(t_{i+p}\right)\right)$ is the total gravitational field at the satellite location at the time $t_{i+p}$ and $\delta \mathbf{e}=\delta \mathbf{e}\left(t_{i+p}\right)$ is the error in the unit vector defining the LOS orientation at the time $t_{i+p}$.

\subsection{Preliminary analysis of actual data noise}

The methodology presented in Sect. 2.2 is applied to produce realizations of noise in the GRACE-based range combinations for 11 months in 2006: from January to November (the month of December is skipped because of a low data quality in the last 8 days of the month). As it is explained in Sect. 2.2.5, each monthly noise realization is obtained by differencing the corresponding sets of the observed and forecasted range combinations. Later on, these realizations are 


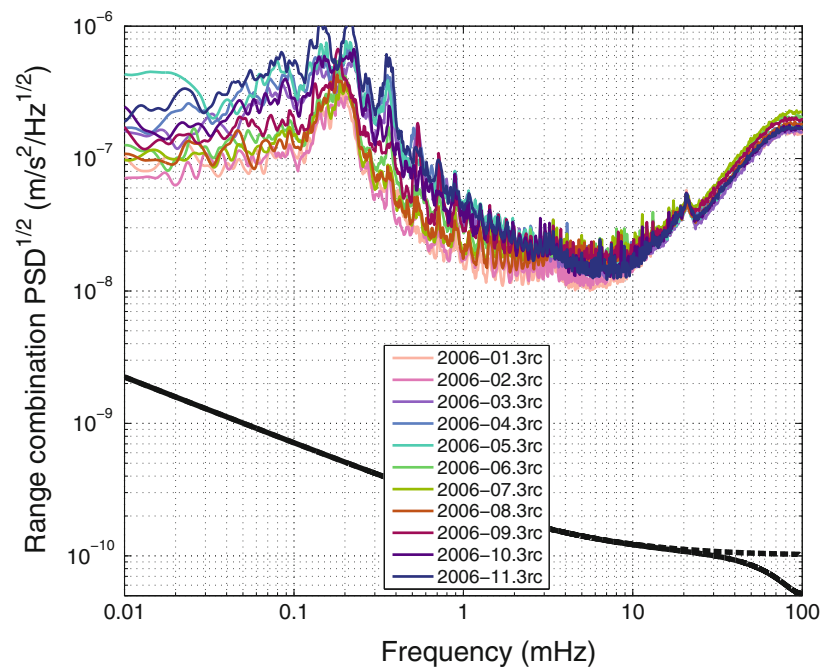

Fig. $2 \mathrm{PSD}^{\frac{1}{2}}$ of the actual realizations of noise in the GRACE-based range combinations for January-November 2006, as well as a theoretical PSD ${ }^{\frac{1}{2}}$ of accelerometer noise directly reproduced from (Kim 2000) (thick black dashed line) and the same PSD ${ }^{\frac{1}{2}}$ transformed into range combinations by multiplying with the averaging filter gain (thick black solid line)

referred to as "actual noise realizations" (in order to distinguish them from synthetic noise realizations that reflect the individual contributions of hypothetical sources to the noise budget). We emphasize that the actual noise realizations reflect inaccuracies both in the observations and in the forecasts, as explained in Sect. 2.1.

In Fig. 2, the spectra of the produced noise realizations are shown in terms of the square root of Power Spectral Density (thereafter, this quantity is denoted as $\mathrm{PSD}^{\frac{1}{2}}$ ). One can see that all the monthly $\mathrm{PSD}^{\frac{1}{2}}$ share a number of common features. First of all, the plot reveals an increased noise level below the frequency of $1 \mathrm{mHz}$. This level shows large variations from month to month and, therefore, can hardly be explained by sensor noise. In Sect. 3, this feature is explained by inaccuracies in the estimated GRACE orbits (more specifically, it is associated with noise of $\delta_{C}$ and $\bar{\delta}_{\mathrm{P}}$ types). Second, all the $\mathrm{PSD}^{\frac{1}{2}}$ show a peak between the 10 and $20 \mathrm{mHz}$. In Sect. 4.1, it is demonstrated that the origin of this feature is noise $\bar{\delta}_{\mathrm{GS}}$ caused by an imperfectness of the exploited static gravity field model. Third, all the $\mathrm{PSD}^{\frac{1}{2}}$ show a rapid increase in the high-frequency range (above $10 \mathrm{mHz}$ ), being practically independent of the month. The likely cause of this feature is ranging noise $\delta_{R}$ related to a limited accuracy of the KBR sensor (Frommknecht et al. 2006; Flury et al. 2008). Noise of this type is further discussed in Sect. 4.2.

Finally, Fig. 2 shows also the theoretical PSD ${ }^{\frac{1}{2}}$ of accelerometer noise (Kim 2000) (thick black dashed line). Strictly speaking, this curve is not directly comparable with the actual noise $\mathrm{PSD}^{\frac{1}{2}}$, since the latter ones are shown in terms or range combinations, which can be interpreted as averaged inter- satellite accelerations (Sect. 2.2.3). In order to eliminate this inconsistency, we have scaled the theoretical PSD ${ }^{\frac{1}{2}}$ of accelerometer noise with the averaging filter gain, the result being shown as thick black solid line. At low and intermediate frequencies, the application of the averaging filter does not have a visible influence, and only at high frequencies (above $30 \mathrm{mHz}$ ) it reduces $\mathrm{PSD}^{\frac{1}{2}}$ up to two times. This comparison shows that in practice there is no substantial difference between a discrete quantity (like a range combination) and its continuous analog, unless frequencies comparable with the Nyquist one are considered.

It is also worth adding that, according to Flury et al. (2008), actual accelerometer noise fits the theoretical expectations only within a few time intervals when the on-board heaters are de-activated. In the rest of the time, the actual accelerometer noise can exceed the theoretical one by an order of magnitude (Frommknecht et al. 2006). But even in that case, accelerometer noise remains much lower than actual one at all frequencies. This means that the on-board GRACE accelerometers provide, most probably, only a minor contribution to the GRACE noise budget. For this reason, the accelerometer noise is not discussed in this paper further.

We would like to stress that the obtained $\mathrm{PSD}^{\frac{1}{2}}$ of actual noise can be of interest not only in the context of the considered functional model, but also in a more general sense. Let us consider, for instance, a commonly used functional model based on range-rates. According to Sect. 2.1, a data noise estimate $\delta \dot{\rho}$ in that case would be equal to

$\delta \dot{\rho}=\delta \dot{\rho}_{\mathrm{O}}-\delta \dot{\rho}_{\mathrm{F}}$,

where $\delta \dot{\rho}_{\mathrm{O}}$ is noise in the observed range-rates, whereas $\delta \dot{\rho}_{\mathrm{F}}$ is noise in the range-rates forecasted on the basis of best possible estimates of GRACE orbits (i.e., the orbits based on best possible force models, best possible estimates of initial state vectors, etc.). The differentiation of Eq. (24) allows noise in terms of range-accelerations to be defined:

$\delta \ddot{\rho}=\delta \ddot{\rho}_{\mathrm{O}}-\delta \ddot{\rho}_{\mathrm{F}}$.

In view of Eq. (7), this expression can be re-written as

$\delta \ddot{\rho}=\delta \ddot{\rho}_{\mathrm{O}}-\delta a_{i}^{(x)}+\delta_{\mathrm{C}}=\left(\delta \ddot{\rho}_{\mathrm{O}}+\delta_{\mathrm{C}}\right)-\delta a_{i}^{(x)}$,

where $\delta_{\mathrm{C}}$ is the correction noise defined by Eq. (17) and $\delta a_{i}^{(x)}$ is noise in the forecasted along-track inter-satellite accelerations, which is the continuous analog of the forecast noise $\delta_{\mathrm{F}}$ given by Eqs. (18) and (19) (i.e., forecast noise in the absence of the averaging filter). Furthermore, the term $\left(\delta \ddot{\rho}_{\mathrm{O}}+\delta_{\mathrm{C}}\right)$ is the continuous analog of observation noise $\delta_{\mathrm{O}}$ given by Eq. (15). This means that at most frequencies, the noise realizations obtained in our analysis are consistent with those in case of a functional model based on range-accelerations. And only in the vicinity of the Nyquist frequency, the finite-difference nature of our functional model should be taken into 
account because it may reduce the apparent noise level up to two times. Finally, a transition of noise in terms of rangeaccelerations into noise in terms of range-rates is straightforward: it is sufficient to multiply the obtained noise PSD ${ }^{\frac{1}{2}}$ with a factor $(2 \pi f)^{-1}$ (such a scaling corresponds to the integration in the time domain).

\section{Inaccuracies in the computed satellite orbits}

In this section, we discuss the contribution of inaccuracies in the computed satellite orbits to the noise budget. The corresponding noise types are identified in Sect. 2.2.6 as correction noise $\left(\delta_{\mathrm{C}}\right)$, satellite position noise $\left(\bar{\delta}_{\mathrm{P}}\right)$, and LOS orientation noise $\left(\bar{\delta}_{\mathrm{L}}\right)$. In the first part of the section, we assess the contribution of orbit inaccuracies analytically, using a number of simplifying assumption. In the second part, we present some results based on an analysis of real GRACE data.

\subsection{Analytic analysis under simplifying assumptions}

The presented analytic analysis has been made to quantify roughly the contribution of state vector errors (taking into consideration different components: along-track, crosstrack, and radial) to the aforementioned noise types and, therefore, to the noise budget in general. The following simplifying assumptions are made:

1. The Earth's gravitation $g(\mathbf{r})$ is described by a central field: $g(\mathbf{r})=\frac{\mathrm{GM}}{\mathbf{r}^{2}}$, where GM is the geocentric gravitational constant;

2. The GRACE satellites follow the same circular orbit, so that the distance between them remains constant.

3. All the errors are described by functions which change in time slowly.

First of all, let us consider correction noise $\delta_{\mathrm{C}}$. According to Eq. (17), it can be represented as

$\delta_{\mathrm{C}}=\delta_{C v}+\delta_{C r}$,

where $\delta_{C v}$ is the contribution of errors in the inter-satellite velocities:

$\delta_{C v}=-\frac{2 v^{(z)}}{\rho} \delta v^{(z)}$

and $\delta_{C r}$ is the contribution of errors in the inter-satellite ranges:

$\delta_{C r}=\frac{\left(v^{(z)}\right)^{2}}{\rho^{2}} \delta \rho$

(note that the lower index $i$ is skipped everywhere in order to simplify the notation). From simple geometrical consider- ations, it follows that

$v^{(z)}=V_{\text {orb }} \frac{\rho}{R_{\text {orb }}}$,

where $R_{\text {orb }}$ is the orbit radius and $V_{\text {orb }}$ is the linear velocity of a satellite in the orbit:

$V_{\mathrm{orb}}=\sqrt{\frac{\mathrm{GM}}{R_{\mathrm{orb}}}}$.

After the substitution of Eqs. (30) and (31) into (28) and (29), we obtain, respectively,

$\delta_{C v}=-2 \sqrt{\frac{\mathrm{GM}}{R_{\mathrm{orb}}^{3}}} \delta v^{(z)}$

and

$\delta_{C r}=\frac{\mathrm{GM}}{R_{\text {orb }}^{3}} \delta \rho$.

Next, let us consider the satellite position noise $\bar{\delta}_{P}$ given by Eqs. (19), (20) and (21). According to our assumptions, noise of all considered types changes in time slowly. Therefore, the application of the averaging filter can be skipped, so that

$\bar{\delta}_{\mathrm{P}}=\left(\mathbf{G}^{(\mathbf{x})}\left(\mathbf{r}_{1}\right) \cdot \delta \mathbf{r}_{1}\right)-\left(\mathbf{G}^{(\mathbf{x})}\left(\mathbf{r}_{2}\right) \cdot \delta \mathbf{r}_{2}\right)$,

where all the quantities are associated with the given time $t_{i}$. Let us introduce individual components of the position vectors $\mathbf{r}_{k}$ and position error vectors $\delta \mathbf{r}_{k}(k=1,2)$ :

$\left\{\begin{array}{l}\mathbf{r}_{k}=\left(x_{k}, y_{k}, z_{k}\right)^{T}, \\ \delta \mathbf{r}_{k}=\left(\delta x_{k}, \delta y_{k}, \delta z_{k}\right)^{T}\end{array}\right.$

see also Fig. 3. In case of a central gravitational field, the vector $\mathbf{G}^{(\mathbf{x})}\left(\mathbf{r}_{k}\right)$ has a simple analytic representation:

$\mathbf{G}^{(\mathbf{x})}\left(\mathbf{r}_{k}\right)=\frac{\mathrm{GM}}{r_{k}^{3}}\left(\frac{3 x_{k}^{2}}{r_{k}^{2}}-1, \frac{3 x_{k} y_{k}}{r_{k}^{2}}, \frac{3 x_{k} z_{k}}{r_{k}^{2}}\right)$,

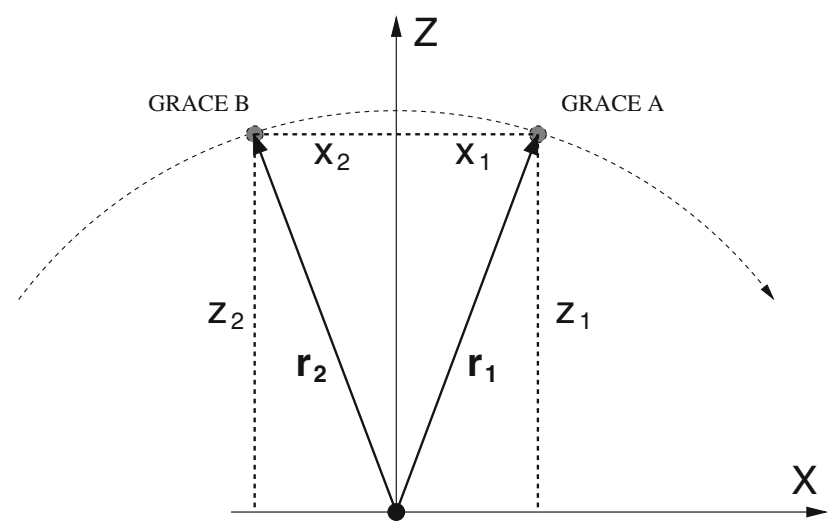

Fig. 3 Position vectors $\mathbf{r}_{k}(k=1,2)$ and their components in the local frame 
where $r_{k}=\sqrt{x_{k}^{2}+y_{k}^{2}+z_{k}^{2}}$. Furthermore, it is clear from Fig. 3 that $r_{1}=r_{2}=R_{\text {orb }} ; x_{1}=-x_{2} ; y_{1}=y_{2}=0$; and $z_{1}=z_{2}$. Then, the satellite position noise is given by

$$
\begin{aligned}
\bar{\delta}_{\mathrm{P}}= & \frac{\mathrm{GM}}{R_{\mathrm{orb}}^{3}}\left[\left(\frac{3 x_{1}^{2}}{R_{\mathrm{orb}}^{2}}-1\right) \delta_{x_{1}}+\frac{3 x_{1} z_{1}}{R_{\text {orb }}^{2}} \delta_{z_{1}}\right] \\
& -\frac{\mathrm{GM}}{R_{\text {orb }}^{3}}\left[\left(\frac{3 x_{1}^{2}}{R_{\mathrm{orb}}^{2}}-1\right) \delta_{x_{2}}-\frac{3 x_{1} z_{1}}{R_{\text {orb }}^{2}} \delta_{z_{2}}\right] \\
= & \frac{\mathrm{GM}}{R_{\text {orb }}^{3}}\left[\left(\frac{3 x_{1}^{2}}{R_{\text {orb }}^{2}}-1\right)\left(\delta_{x_{1}}-\delta_{x_{2}}\right)+\frac{3 x_{1} z_{1}}{R_{\text {orb }}^{2}}\left(\delta_{z_{1}}+\delta_{z_{2}}\right)\right] .
\end{aligned}
$$

Let us introduce the error $\delta z_{m}$ in the z-coordinate of the middle point between the satellites:

$\delta z_{m}=\frac{1}{2}\left(\delta_{z_{1}}+\delta_{z_{2}}\right)$.

Furthermore, let us notice that $\left(\delta_{x_{1}}-\delta_{x_{2}}\right)$ is nothing but the error in the inter-satellite range estimated on the basis of the computed GRACE orbits. We denote this error as $\delta x$, to distinguish it from the error $\delta \rho$ in the ranges measured with the KBR sensor, which was introduced earlier.

In addition, we take into account that $x_{1}=-x_{2}=\frac{1}{2} \rho$; $\frac{3 x_{1}^{2}}{R_{\text {orb }}^{2}} \ll 1$; and $z_{1} \approx R_{\text {orb }}$. Then, we finally obtain

$\bar{\delta}_{\mathrm{P}}=\bar{\delta}_{P r}+\bar{\delta}_{P a}$,

where

$\bar{\delta}_{P r}=-\frac{\mathrm{GM}}{R_{\text {orb }}^{3}} \delta x$

is the contribution of relative positioning errors and

$\bar{\delta}_{P a}=\frac{3 \mathrm{GM} \rho}{R_{\text {orb }}^{4}} \delta z_{m}$

is the contribution of absolute positioning errors to the positioning noise $\bar{\delta}_{\mathrm{P}}$.

At last, let us consider the LOS orientation noise $\bar{\delta}_{\mathrm{L}}$ given by Eqs. (19), (22) and (23). In the absence of the averaging filter, we have

$\bar{\delta}_{\mathrm{L}}=\bar{\delta}_{L_{1}}-\bar{\delta}_{L_{2}}$,

where

$\bar{\delta}_{L_{k}}=\left(\mathbf{g}\left(\mathbf{r}_{\mathbf{k}}\right) \cdot \delta \mathbf{e}\right) \quad(k=1,2)$,

all the quantities being associated with the given time $t_{i}$. Let us introduce the component-wise notation for the LOS orientation vector $\delta \mathbf{e}: \delta \mathbf{e}=\left(\delta e^{(x)}, \delta e^{(y)}, \delta e^{(z)}\right)^{T}$. Then, the contribution of the leading satellite to the LOS orientation noise can be written as

$$
\begin{aligned}
\bar{\delta}_{L_{1}} & =\left(\mathbf{g}\left(\mathbf{r}_{1}\right) \cdot \delta \mathbf{e}\right) \\
& =g^{(x)}\left(\mathbf{r}_{1}\right) \delta e^{(x)}+g^{(y)}\left(\mathbf{r}_{1}\right) \delta e^{(y)}+g^{(z)}\left(\mathbf{r}_{1}\right) \delta e^{(z)} .
\end{aligned}
$$

Since the vector $\mathbf{e}$ in the local frame is equal to $(1 ; 0 ; 0)^{T}$ and the vector $\delta \mathbf{e}$ is orthogonal to it, $\delta e^{(x)}=0$. Furthermore, $g^{(y)}\left(\mathbf{r}_{1}\right)=0$ in a central field. Therefore,

$\bar{\delta}_{L_{1}}=g^{(z)}\left(\mathbf{r}_{1}\right) \delta e^{(z)}$

and, similarly,

$\bar{\delta}_{L_{2}}=g^{(z)}\left(\mathbf{r}_{2}\right) \delta e^{(z)}$.

In addition, the $\mathrm{z}$-component of the central gravitational field is equal at the satellite locations to

$g^{(z)}\left(\mathbf{r}_{1}\right)=g^{(z)}\left(\mathbf{r}_{2}\right)=-\frac{\mathrm{GM} z_{1}}{R_{\text {orb }}^{3}}$.

Consequently, we finally arrive at

$\bar{\delta}_{\mathrm{L}}=\bar{\delta}_{L_{1}}-\bar{\delta}_{L_{2}}=0$.

In other words, the LOS orientation error vanishes under the assumptions made.

In summary, the conducted analytic analysis allows us to identify the following primary contributors to the noise budget that are caused by errors in the estimated satellite orbits:

- contributor $\delta_{C v}$ caused by the error $\delta v^{(z)}$ in the radial component of the inter-satellite velocities, Eq. (32);

- contributor $\bar{\delta}_{P r}$ caused by the error $\delta x$ in the along-track component of the inter-satellite offset vector (i.e., the inter-satellite range) derived from the computed GRACE orbits, Eq. (39);

- contributor $\bar{\delta}_{P a}$ caused by the error $\delta z_{m}$ in the radial component of the inter-satellite mid-point position (i.e., roughly speaking, the mean altitude of the two satellites), Eq. (40).

In addition, we have identified the contributor $\delta_{C r}$ caused by the inter-satellite ranging error $\delta \rho$, Eq. (33), propagated into the estimated centrifugal accelerations; this contributor must not be confused with ranging noise $\delta_{\mathrm{R}}$, Eq. (16).

Let us make a rough assessment of these four contributors quantitatively. To that end, we need to introduce realistic standard deviations (std) of the errors $\delta v^{(z)}, \delta x, \delta z_{m}$, and $\delta \rho$.

The std of the error $\delta v^{(z)}$ can be easily estimated if we assume that all three components of the inter-satellite velocities derived from GRACE orbits are equally accurate. In that case, it is sufficient to compare the inter-satellite velocities derived from the computed orbits with the range-rates measured with the KBR sensor. The conducted comparison showed that errors in the inter-satellite velocities derived from the estimated GRACE orbits are characterized, in average, by the std of about $4 \mu \mathrm{m} / \mathrm{s}$. Details of this numerical study are presented in the next subsection.

The std of the errors $\delta x$ can be estimated in at least two different ways. The first way is similar to that mentioned above, i.e., to compare the inter-satellite distances derived 
from the computed GRACE orbits with those based on the KBR measurements. It shows that the level of the errors under consideration is, in average, about $4 \mathrm{~mm}$. The second way is to estimate this std on the basis of errors in inter-satellite velocities, assuming that the latter are periodic. This idea is based on the fact that range errors described by the function

$\delta x(t)=C \sin \left(\frac{2 \pi t}{T}\right)$

lead to range-rate errors given by the function

$\delta v^{(x)}(t)=\frac{2 \pi C}{T} \cos \left(\frac{2 \pi t}{T}\right)$,

where $T$ is the period and $C$ is a certain constant.

A comparison of Eqs. (48) and (49) leads to the following relationship:

$\operatorname{std}[\delta x]=\operatorname{std}\left[\delta v^{(x)}\right] \cdot \frac{T}{2 \pi}$.

Analysis of the GRACE orbits showed that the dominant period of the range errors is about $5,400 \mathrm{~s}$ (1 revolution). Then, Eq. (50) allows std $[\delta x]$ to be estimated as about $4 \mathrm{~mm}$, which is consistent with the result obtained in the first way. This can be considered as a confirmation of our assumption that the errors in the radial and along-track component of inter-satellite velocities stay at the same level.

The std of the error $\delta z_{m}$ cannot be estimated on the basis of KBR data at all. In our analysis, we set it equal to $2 \mathrm{~cm}$. This value is similar to that observed in a GRACE orbit validation based on a comparison with SLR data (Q. Zhao, personal communication).

Finally, the std of the error $\delta \rho$ (i.e. the accuracy of KBR data) is set equal to $1 \mu \mathrm{m}$.

The remaining values needed for the intended quantitative estimation are the geocentric gravitational constant GM ( $\approx 3.986 \times 10^{14} \mathrm{~m}^{3} \mathrm{~s}^{-2}$ ); the orbit radius, $R_{\text {orb }}$, which is set equal to $6,850 \mathrm{~km}$, and the inter-satellite range $\rho(\approx 200 \mathrm{~km})$.

After inserting all these numbers into Eqs. (32), (39), (40), and (33), we obtain, respectively,

$$
\left\{\begin{array}{l}
\operatorname{std}\left[\delta_{C v}\right]=9 \times 10^{-9} \mathrm{~m} / \mathrm{s}^{2} \\
\operatorname{std}\left[\bar{\delta}_{P r}\right]=5 \times 10^{-9} \mathrm{~m} / \mathrm{s}^{2} \\
\operatorname{std}\left[\bar{\delta}_{P a}\right]=7 \times 10^{-10} \mathrm{~m} / \mathrm{s}^{2} \\
\operatorname{std}\left[\delta_{C r}\right]=1 \times 10^{-12} \mathrm{~m} / \mathrm{s}^{2}
\end{array}\right.
$$

Noise of such a level (especially, in the first two cases) definitely may not be negligible. A more in-depth analysis requires, however, that noise of a particular type is quantified not only by just a single number (standard deviation), but also by PSD ${ }^{\frac{1}{2}}$, which shows the noise level in different frequency ranges. This is done in the next subsection on the basis of real data.

\subsection{Analysis based on real data}

In this subsection, we present a spectral analysis of two contributors to the noise budget:

- Noise $\delta_{C v}$ caused by an inaccurately estimated radial component of the inter-satellite velocities;

- Noise $\bar{\delta}_{P r}$ caused by inaccuracies in the inter-satellite ranges derived from the computed GRACE orbits.

As shown in the previous subsection, these two contributors can be especially significant among all related to inaccuracies in the computed orbits. This time, our analysis is based on real GRACE data (namely, KBR measurements and dynamic orbits), which have been already presented in Sect. 2 .

Synthetic realizations of noise $\delta_{C v}$ are generated as follows: first of all, we produce time series of the along-track inter-satellite velocities by applying a multi-point differentiation to the dynamic orbits of GRACE satellites. Then, we generate the errors in these time series by subtracting the precise range-rates, which are measured with the KBR sensor. The resulting error time series for July 2006 is shown in Fig. 4a, whereas a 6-hour fragment of this time series is presented in Fig. 4b. The std of the obtained difference in July is $2.2 \mu \mathrm{m} / \mathrm{s}$; for other months in 2006, it varies between 2.0 and $7.2 \mu \mathrm{m} / \mathrm{s}$ (on average, $4.0 \mu \mathrm{m} / \mathrm{s}$ ). The obtained time series is
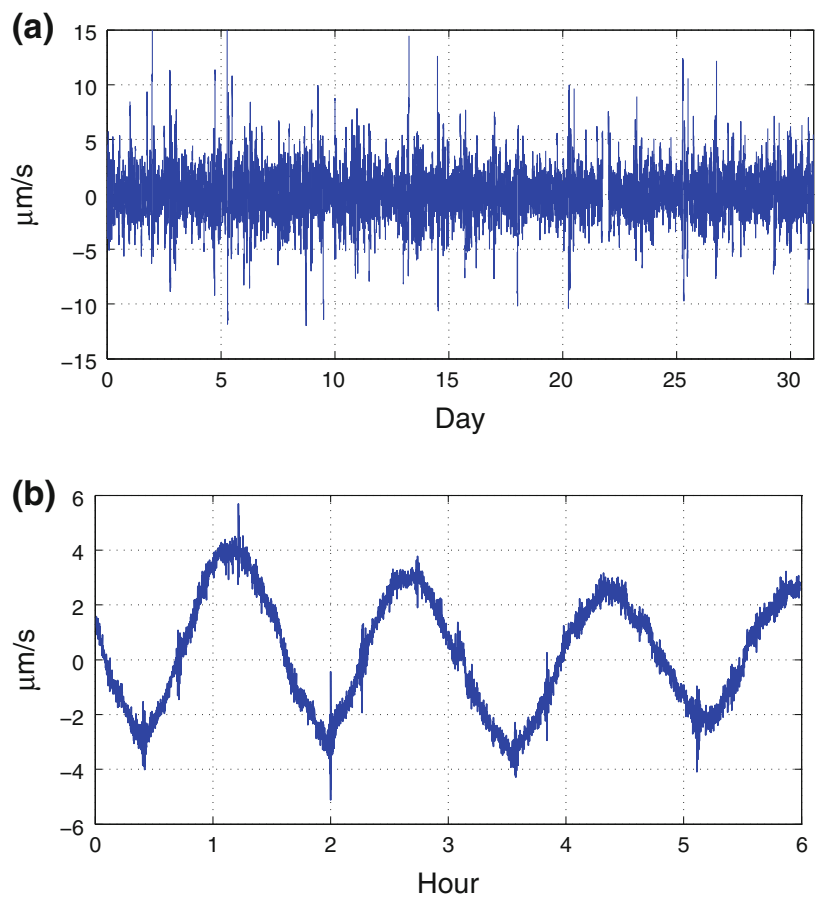

Fig. 4 Difference between the range-rates derived from the computed GRACE orbits and those based on KBR measurements: a as a time series for the entire month of July 2006 and $\mathbf{b}$ as a 6-h fragment of the time series shown in the top plot 
characterized by a quasi-periodic behavior with a dominant period close to $1.5 \mathrm{~h}$. Since noise in the range-rates based on KBR measurements is mostly concentrated in the range of high frequencies, we conclude that the produced difference mostly reflects noise associated with satellite orbits. At the same time, we notice that this difference may not be totally free from KBR-related errors. In particular, those errors are the most probable cause of high-frequency variations clearly visible in Fig. $4 \mathrm{~b}$.

To make the second step, we assume that the stochastic properties of noise in the along-track and in the radial component of orbit-based inter-satellite velocities are similar. This allows us to produce realizations of noise $\delta_{C v}$ using Eq. (28), where the term $\delta v^{(z)}$ is replaced with $\delta v^{(x)}$. The $\operatorname{PSD}^{\frac{1}{2}}$ of the produced noise realization for July 2006 is shown in Fig. 5 in green. The $\mathrm{PSD}^{\frac{1}{2}}$ of the actual noise is also shown in this figure for a reference (red curve). One can see that the two $\mathrm{PSD}^{\frac{1}{2}}$ nearly coincide with each other in the frequency range from 0.1 to $1 \mathrm{mHz}$. A similar consistency is observed also for the other months (not shown). This can be interpreted as a very strong evidence that the $\delta_{C v}$ contributor to the error budget is dominant in the aforementioned frequency range. On the other hand, the considered contributor rapidly diminishes at the frequencies above $1 \mathrm{mHz}$ (i.e., at the periods shorter than $1,000 \mathrm{~s})$. Therefore, the high-frequency variations visible in Fig. 4b, which are presumably not related to inaccuracies in the computed orbits, do not play a significant role in the conducted analysis.

To produce realizations of noise $\bar{\delta}_{P r}$, we apply the following procedure: first of all, we prepare "adjusted" GRACE orbits, using the available dynamic orbits and the de-biased

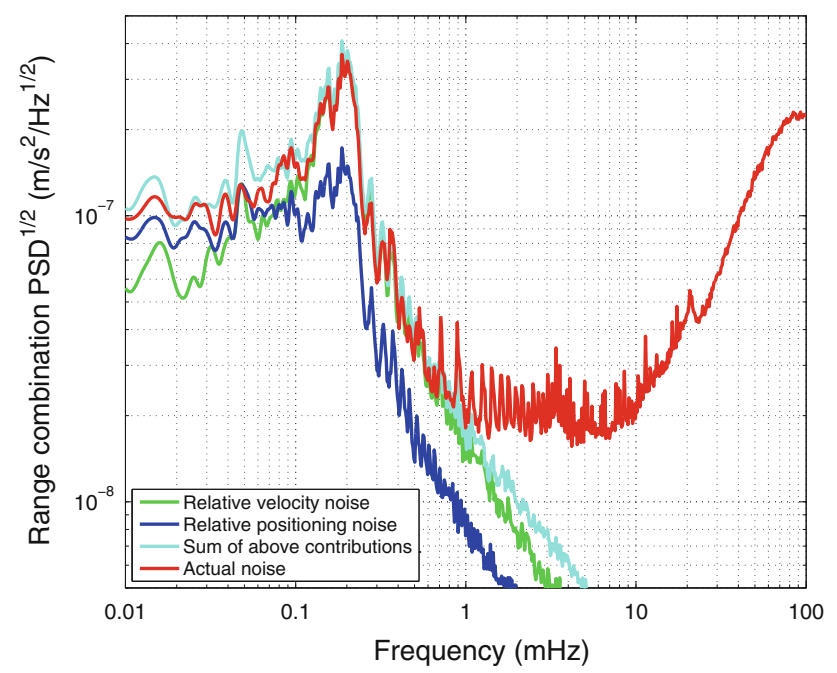

Fig. $5 \operatorname{PSD}^{\frac{1}{2}}$ of noise in GRACE data: actual noise (in red); synthesized contributor $\delta_{C v}$ that reflects inaccuracies in the radial component of the orbit-derived inter-satellite velocities (in green); contributor $\bar{\delta}_{P r}$ that reflects inaccuracies in the orbit-derived ranges (in blue); and the sum of $\delta_{C v}$ and $\bar{\delta}_{P r}$ (in cyan). The considered month is July, 2006
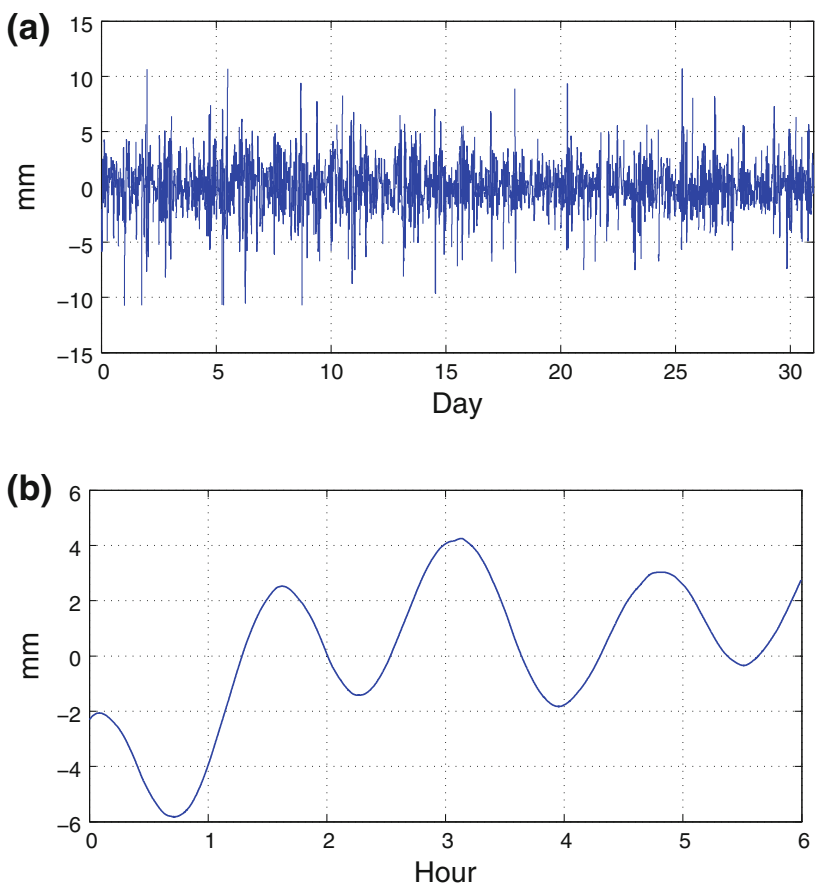

Fig. 6 Difference between the ranges derived from the computed GRACE orbits and those based on KBR measurements: a as a time series for the entire month of July 2006 and $\mathbf{b}$ as a 6-h fragment of the time series shown in the top plot

KBR measurements. The adjusted orbits are characterized by the same positions of inter-satellite mid-points and the same LOS vectors. The inter-satellite ranges, however, are forced to be equal to those measured with the KBR sensor. To quantify this adjustment, we have produced time series of the difference $\delta_{x}$ between the ranges based on the dynamic orbits and those from the KBR data set. The results for July 2006 is shown in Fig. 6a, whereas a 6-h fragment of that time series is presented in Fig. 6b. The std of those differences in July 2006 is $2.1 \mathrm{~mm}$; for other months in 2006, it is in the range between 1.8 and $7.0 \mathrm{~mm}$ (on average, $3.7 \mathrm{~mm}$ ). This is about 3 orders of magnitude larger than the noise level of KBR data. Thus, there is no doubt that the origin of the observed differences is noise in the computed orbits.

Further computations are performed in line with the discussion in Sect. 2.2.6. More specifically, we evaluate the force model along the orbits of both types to produce the 3-D accelerations of both GRACE satellites and, consequently, the 3-D inter-satellite accelerations. After that, the averaging filter is applied and the results are projected onto the LOS, cf. Eq. (19). The difference between the time series based on the dynamic and the adjusted orbits is a realization of positioning noise $\bar{\delta}_{P}$ produced under the assumption that the errors $\delta_{x_{1}}$ and $\delta_{x_{2}}$ in the orbit-derived satellite positions are, up to the sign, equal to each other $\left(\delta_{x_{1}}=-\delta_{x_{2}}\right)$. It is important to add that the impact of these errors is pro- 
portional to the $\mathrm{xx}$-component of the matrix of gravitational gradients at the satellite locations, Eq. (21). Since this matrix is close to that of the central field and the $\mathrm{x}$-coordinates of the satellites in the local frame are (up to the sign), approximately equal, the aforementioned gravitational gradient component is approximately the same at the location of the leading and trailing satellite, cf. Eq. (36). This means that the positioning noise $\bar{\delta}_{P}$ is almost insensitive to the proportion in which the error $\delta_{x}$ is split between the trailing and leading satellite.

The PSD ${ }^{\frac{1}{2}}$ of the noise realization produced for July 2006 is shown in the aforementioned Fig. 5 in blue. Noise under consideration, just like noise $\delta_{C v}$, occupies the range of low frequencies, though its magnitude is about two times smaller (only at frequencies below $0.1 \mathrm{mHz}$ it becomes somewhat larger). Figure 5 presents also the PSD ${ }^{\frac{1}{2}}$ of the sum $\bar{\delta}_{P r}+\delta_{C v}$, which nearly coincides with the $\operatorname{PSD}^{\frac{1}{2}}$ of $\delta_{C v}$ and is very close to the PSD ${ }^{\frac{1}{2}}$ of actual noise in the range $0.1-1 \mathrm{mHz}$. At the frequencies below $0.1 \mathrm{mHz}$, the sum $\bar{\delta}_{P r}+\delta_{C v}$ even shows a level exceeding that the actual noise. This might be explained by a systematic phase shift between errors in the radial and the along-track component of orbit-derived intersatellite velocities, which makes the result of the summation somewhat inadequate when the magnitude of the contributors $\bar{\delta}_{P r}$ and $\delta_{C v}$ is similar.
The consideration of other months in 2006 leads to very similar results (not shown), allowing us to conclude that the combination of noise of the two considered types is, most probably, the major factor explaining low-frequency noise in real GRACE data.

From the discussion above, it follows that the presented procedure for computing noise $\bar{\delta}_{\operatorname{Pr}}$ results not only in a stochastic description of this noise, but also allows a time-series to be obtained which is close to that of this noise itself. This means that noise of this type can be, in principle, eliminated when a gravity field model is computed on the basis of GRACE-based range combinations. Our experience tells, however, that the quality of gravity field models changes little after applying this operation. In view of Fig. 5, such an outcome is not surprising: this correction may reduce noise noticeably only at very low frequencies, which play practically no role in gravity field modeling.

The adopted procedures for computing noise realizations of $\delta_{\mathrm{C}}$ and $\bar{\delta}_{P r}$ type are summarized in the top part of Table 2.

\section{Other sources of noise}

In this section we investigate other sources of noise, which play (or may play) a role at the intermediate and high

Table 2 Synthetic noise realizations: types, associated frequency ranges and summary of the procedures to generate them

\begin{tabular}{|c|c|c|c|}
\hline Noise type & $\begin{array}{l}\text { Frequency } \\
\text { range } \\
(\mathrm{mHz})\end{array}$ & Input & Procedure \\
\hline $\begin{array}{l}\text { Noise } \bar{\delta}_{P r} \text { caused by inaccuracies in the } \\
\text { inter-atellite ranges derived from the } \\
\text { computed orbits }\end{array}$ & $\lesssim 0.1$ & $\begin{array}{l}\text { Dynamic orbits } \\
\text { De-biased ranges measured with } \\
\text { the KBR sensor }\end{array}$ & $\begin{array}{l}\text { Producing the adjusted orbits } \\
\text { Computation of 3-D point-wise } \\
\text { inter-satellite accelerations along the } \\
\text { dynamic and adjusted orbits } \\
\text { Application of the averaging filter (cf. Eq. 6) } \\
\text { Projecting onto the LOS } \\
\text { Differencing the results based on the } \\
\text { dynamic and adjusted orbits }\end{array}$ \\
\hline $\begin{array}{l}\text { Noise } \delta_{C v} \text { caused by an inaccurately } \\
\text { estimated radial component of the } \\
\text { inter-satellite velocities }\end{array}$ & $0.1-1$ & $\begin{array}{l}\text { Dynamic orbits } \\
\text { Range-rates measured with the } \\
\text { KBR sensor }\end{array}$ & $\begin{array}{l}\text { Differencing the orbit-based and measured } \\
\text { range-rates } \\
\text { Propagating the obtained errors into } \\
\text { centrifugal accelerations, Eq. }(28)\end{array}$ \\
\hline $\begin{array}{l}\text { Noise } \bar{\delta}_{\mathrm{GV}} \text { caused by errors in } \\
\text { time-varying gravity field models }\end{array}$ & $1-3$ & $\begin{array}{l}10 \% \text { of residual AOD1B signal } \\
\text { or } \\
\text { Residual DMT-1 signal }\end{array}$ & $\begin{array}{l}\text { Computation of 3-D point-wise residual } \\
\text { inter-satellite accelerations } \\
\text { Application of the averaging filter (cf. Eq. 6) } \\
\text { Projecting onto the LOS }\end{array}$ \\
\hline $\begin{array}{l}\text { Empirically defined stationary random } \\
\text { noise }\end{array}$ & $1-9$ & $\mathrm{PSD}^{1 / 2}$ of corrected actual noise & $\begin{array}{l}\text { Generation of stationary random noise to } \\
\text { reproduce the } \operatorname{PSD}^{1 / 2} \text { of corrected actual } \\
\text { noise } \\
\text { Band-pass filtering (band } 1-9 \mathrm{mHz} \text { ) }\end{array}$ \\
\hline $\begin{array}{l}\text { Residual signal due to errors in the } \\
\text { adopted static field model } \\
\text { EIGEN-GL04C }\end{array}$ & $9-23$ & $\begin{array}{l}\text { Static field correction to degree } \\
180 \\
\text { Dynamic orbits }\end{array}$ & $\begin{array}{l}\text { Computation of 3-D point-wise residual } \\
\text { inter-satellite accelerations } \\
\text { Application of the averaging filter (cf. Eq. 6) } \\
\text { Projecting onto the LOS }\end{array}$ \\
\hline Ranging noise $\delta_{\mathrm{R}}$ & $>23$ & $\begin{array}{l}\text { Random frequency-dependent } \\
\text { errors in ranges (Fig. 12a) }\end{array}$ & Double numerical differentiation, Eq. (16) \\
\hline
\end{tabular}


frequencies. In doing so, we compare the actual noise in range combinations with synthetic noise realizations associated with various hypothetical sources. More specifically, three sources of noise are addressed:

- Errors in the static gravity field model, which is a part of the set of background force models

- Ranging sensor errors

- Inaccuracies of models of temporal gravity field variations (associated with mass transport at the Earth's surface)

In addition, a hypothesis is considered that the dominant noise in the frequency range $1-9 \mathrm{mHz}$ is just random stationary noise (of an undefined origin). Considered types of synthetic noise and procedures to generate them are summarized in the bottom part of Table 2 .

As already demonstrated above, spectral analysis is a powerful tool for a comparison of noise realizations. Unfortunately, this tool may not be sufficiently informative if noise of a particular type is associated with processes or features at the Earth surface, so that it manifests itself in the spatial domain rather than in the time or frequency domain. This is the reason why the second way is also followed: to propagate noise into gravity field parameters (spherical harmonic coefficients) and then to analyze it in the spatial domain. In doing so, we apply (in most cases) a band-pass filtering to noise time series in order to highlight the frequency band where noise of a certain type is presumably dominant. Technically, filtering is performed with the 7-th order Butterworth bandpass filter, for which purpose the MATLAB function butterworth is exploited. The filter is applied twice, in the forward and reverse direction, which is equivalent to using a zerophase filter, so that filtered signal is not subject to any time shift. PSD ${ }^{\frac{1}{2}}$ of actual noise obtained after various band-pass filtering is shown in Fig. 7.

Computation of gravity field parameters without a bandpass filtering is considered as well, so that the results could be interpreted as a reproduction of noise that is present in actual GRACE-based gravity field solutions. In that case, we clean the realizations of actual noise from the effects of inaccurately known orbits, which manifest themselves as an increased noise at low frequencies (see Sect. 3). To that end, we use the same scheme as was applied by Liu et al. (2010) in producing the DMT-1 model. A noise time series under consideration is fitted by the analytic function

$$
\begin{aligned}
n(t)= & A+B t+C t^{2}+D \sin \left(\frac{2 \pi t}{T_{\mathrm{rev}}}\right)+E \cos \left(\frac{2 \pi t}{T_{\mathrm{rev}}}\right) \\
& +F t \sin \left(\frac{2 \pi t}{T_{\mathrm{rev}}}\right)+G t \cos \left(\frac{2 \pi t}{T_{\mathrm{rev}}}\right),
\end{aligned}
$$

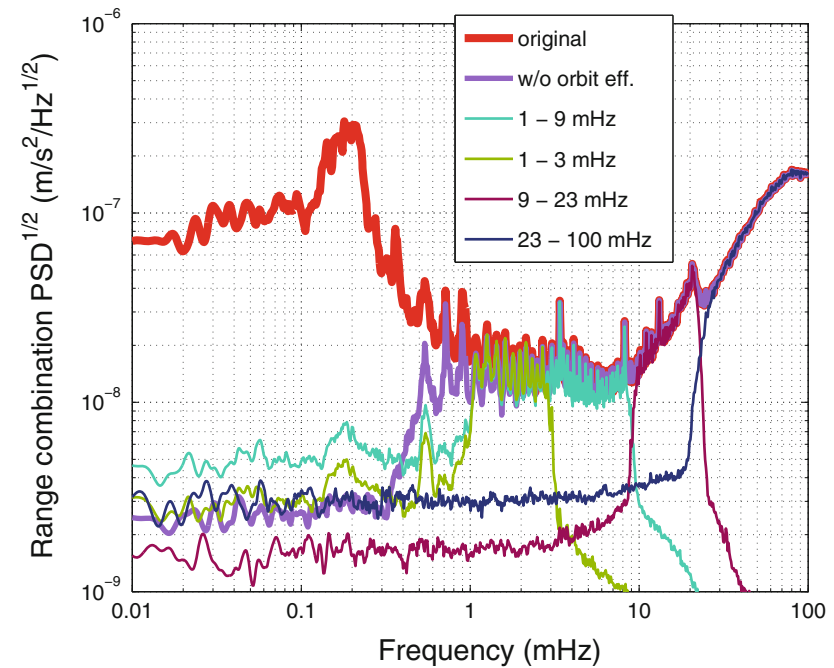

Fig. $7 \mathrm{PSD}^{\frac{1}{2}}$ of actual noise obtained after band-pass filtering applied in order to highlight one of the four frequency bands indicated in the bottom part of Table 2. PSD ${ }^{\frac{1}{2}}$ of original actual noise (red curve) and of noise cleaned from the orbit inaccuracies (purple curve) are also shown. The considered month is February, 2006

where $T_{\text {rev }}$ is the revolution period (approximately $1.5 \mathrm{~h}$ ), and $A, B, C, D, E, F$, and $G$ are constant coefficients computed by means of a least-squares adjustment. This analytic function, which is estimated once per revolution, is subtracted from the original time series.

The inversion procedure used to propagate noise time series into gravity field parameters is also somewhat similar to that designed for the production of the DMT-1 model (Liu et al. 2010). We estimate gravity field parameters by solving a corresponding system of linear equations. The minimum spherical harmonic degree is set equal to 2 , and the maximum one is typically set equal to 120 . Unlike Liu et al. (2010), we apply the frequency-dependent data weighting (Klees et al. 2003; Klees and Ditmar 2004) based on the actual noise PSD $^{\frac{1}{2}}$ (red curve in Fig. 7). This is consistent with the statistically optimal approach to actual gravity field modeling on the basis of GRACE range combinations.

Finally, the integrated effect of the errors of all the considered types onto a recovered gravity field is presented and compared with that of the actual noise.

\subsection{Contribution of errors in the static gravity field model}

In this subsection, the contribution of errors in the static gravity field model to the noise budget is analyzed. As it will become clear later, those errors manifest themselves mostly in the frequency band between 9 and $23 \mathrm{mHz}$. This band, therefore, is the primary focus of our discussion.

To begin with, we consider the hypothesis that actual noise in the aforementioned frequency band can be considered as purely random. We synthesize monthly realizations of a syn- 


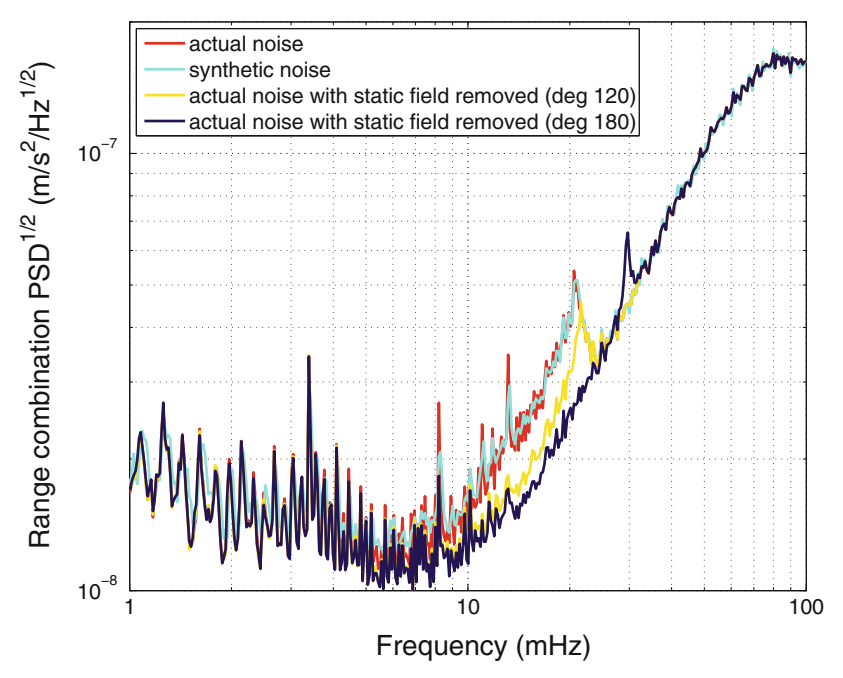

Fig. $8 \mathrm{PSD}^{\frac{1}{2}}$ computed on the basis of the following noise types: actual noise (red line); synthetic stationary noise (light blue line); actual noise corrected for the contribution of the residual static gravity field estimated up to maximum degree 120 (yellow line); and the same, but for the maximum degree 180 (dark blue line). The month under consideration is February, 2006

thetic random stationary noise in such a way that the $\mathrm{PSD}^{\frac{1}{2}}$ of a particular realization coincides with the $\mathrm{PSD}^{\frac{1}{2}}$ of the actual noise in the corresponding month (see the light blue and red curve in Fig. 8, respectively). It is important to mention that actual noise realizations contain gaps due to, e.g., the elimination of outliers from the data (Liu et al. 2010). All such gaps are fully reproduced in the realizations of synthetic noise.

Noise realizations of both types - actual and syntheticare band-pass filtered (band 9-23 $\mathrm{mHz}$ ) and propagated into gravity field parameters. It turns out that the resulting RMS geoid height error in case of the actual noise is more than 2 times larger than in case of the synthetic noise for all the months (see the red and light blue curve in Fig. 9, respectively). We interpret this observation as an evidence that the actual noise is characterized by correlations in the spatial domain, which cannot be described by the $\mathrm{PSD}^{\frac{1}{2}}$ and, therefore, are not reproduced in the synthetic noise. To reveal the nature of these spatial correlations, we compute the mean value of the gravity field parameters over the considered 11 months. It turns out that the resulting RMS geoid height is $6.9 \mathrm{~cm}$, i.e., close to the mean monthly RMS value, $7.5 \mathrm{~cm}$ (the mean level of the red curve in Fig. 9). This is in contrast to the case of the synthetic noise, where the RMS geoid height of the 11-month mean field is only $1 \mathrm{~cm}$, which is about $\sqrt{11}$ times smaller than the mean monthly RMS value, $3.3 \mathrm{~cm}$ (the mean level of the light blue curve in Fig. 9), which is fully consistent with the statistical theory. This allows us to suggest that the observed phenomenon is nothing but an evidence of residual static signal left in the noise realizations due to inaccuracies in the exploited static gravity model (namely, EIGEN-GL04C).

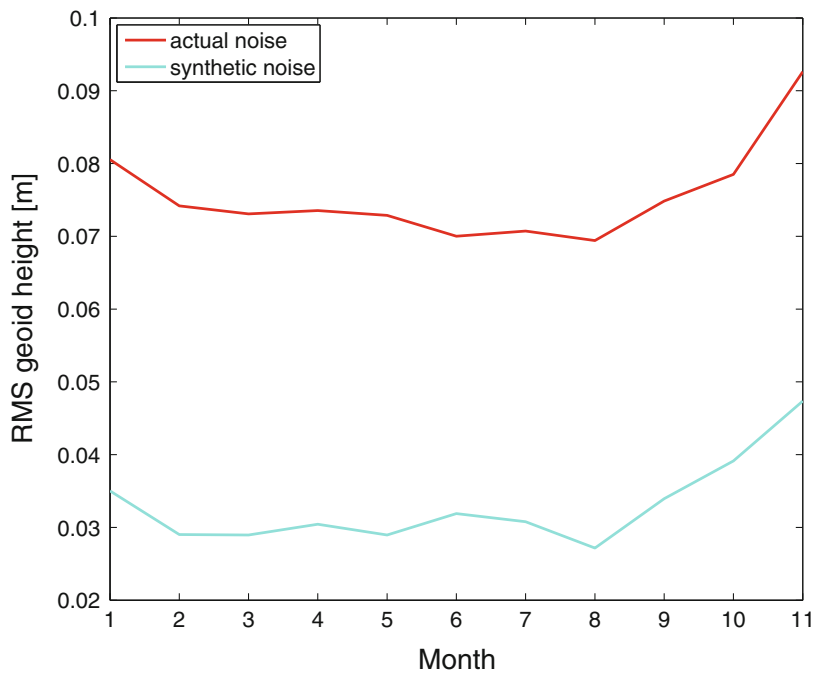

Fig. 9 RMS geoid height error computed after the propagation of band-pass filtered monthly noise realizations into gravity field parameters (as a function of month). Considered noise types: actual noise (red line) and synthetic stationary noise (light blue line)

In order to confirm that we do the following: the procedure described above is repeated without applying a band-pass filter, and the produced 11-month mean gravity field is used as a correction to the EIGEN-GL04C model. On the basis of this correction, residual range combinations are computed as explained in Sect. 2.2, cf. Eq. (6). Then, the residual range combinations are subtracted from the original realizations of actual noise. The $\mathrm{PSD}^{\frac{1}{2}}$ of a corrected noise realization is shown in Fig. 8 in yellow. One can see that the updated noise is indeed noticeably lower in the frequency band between 9 and $23 \mathrm{mHz}$. Thus, inaccuracies of the exploited static gravity field model are one of the most important contributors to the noise budget in the aforementioned frequency range. In other words, the observed noise, which is rapidly increasing above $9 \mathrm{mHz}$, is (at least, partly) explained by the presence of residual gravity field signal in the range combinations, which is left after the subtraction of imperfectly forecasted range combinations.

At the first glance, such a conclusion is counterintuitive. It is well known that gravity field signal observed at the satellite altitude decreases as the frequency increases due to the upward continuation effect. One should keep in mind, however, that we currently deal with a very specific type of a residual signal: the signal caused by the difference between the true mean gravity field and the one described by the EIGENGL04C model, i.e., the residual signal that is equal to the errors in the latter model. Those errors increase with spherical harmonic degree and so does the residual signal. Apparently, this increase compensates the upward continuation effect.

It is also interesting to see that the corrected noise $\mathrm{PSD}^{\frac{1}{2}}$ still contains a peak near the frequency of $20 \mathrm{mHz}$ or 120 cycles-per-revolution (cpr). This peak is explained by the 
presence of residual static field signal above degree 120. In order to demonstrate that we repeat the noise correction procedure, having re-computed the mean field to degree 180 . The resulting $\operatorname{PSD}^{\frac{1}{2}}$ is shown in Fig. 8 in dark blue. One can see that the peak at $20 \mathrm{mHz}$ has vanished, but a new (though smaller) peak around the frequency of $33 \mathrm{mHz}$ (or $180 \mathrm{cpr}$ ) has appeared. Thus, even a gravity field model complete to degree 180 cannot fully explain all the signal present in GRACE data.

Finally, we analyze whether the computed correction may be considered as an actual improvement of the EIGEN-GL04C model (in other words, whether this correction is close to the difference between the true field and the one described by the EIGEN-GL04C model). To this end, we compare the computed correction with the residual field defined as the difference between the state-of-the-art GOCO02S model (Goiginger et al. 2011) and the EIGENGL04C model, truncation at degree 180 being applied. It turns out that the similarity between the two residual fields is not obvious (not shown). In order to clean the computed correction from noise and nuisance signals, we return to the correction presented above (maximum degree is 120; bandpass filter is applied to the actual noise realizations in order to highlight the range 9-23 mHz). Furthermore, the difference between the GOCO02S and the EIGEN-GL04C model is also truncated at degree 120. After that, a visual comparison of the two maps of residual geoid heights shows that some similarity between them indeed exists, but only in the polar areas (see, e.g., Fig. 10).

In order to make a more objective comparison of the two residual fields, we estimate a correlation between them as a function of latitude and longitude. The procedure is the following: the two functions to be compared are computed on the equiangular $1.5^{\circ} \times 1.5^{\circ}$ grid. Then, a fragment of the grid of a certain size $2 k+1$ by $2 k+1$ is selected, the correlation coefficient between the two functions within the selected fragment is computed, and then assigned to the node at the center of the fragment. The procedure is repeated until a global grid of correlation coefficients is obtained. Unfortunately, the spatial pattern shown in such a grid turns out to be closely related to the parameter $k$. The produced spatial correlation map is strongly dominated by features of the size of $k$ cells, i.e., in general, not sufficiently representative. In order to eliminate a dependence on a pre-defined fragment size, we produce multiple correlation coefficient grids, changing the parameter $k$ uniformly from 1 to 30 , and then compute the mean of all of these grids. The obtained result is shown in Fig. 11. This map confirms a high correlation between the two residual fields in the polar regions and a lower correlation at low latitudes.

We explain such an outcome by the fact that the difference between the azimuths of the ascending and descending (a)

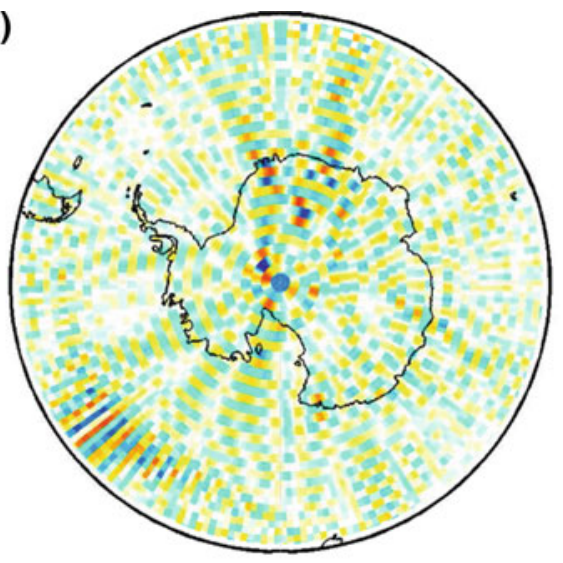

(b)
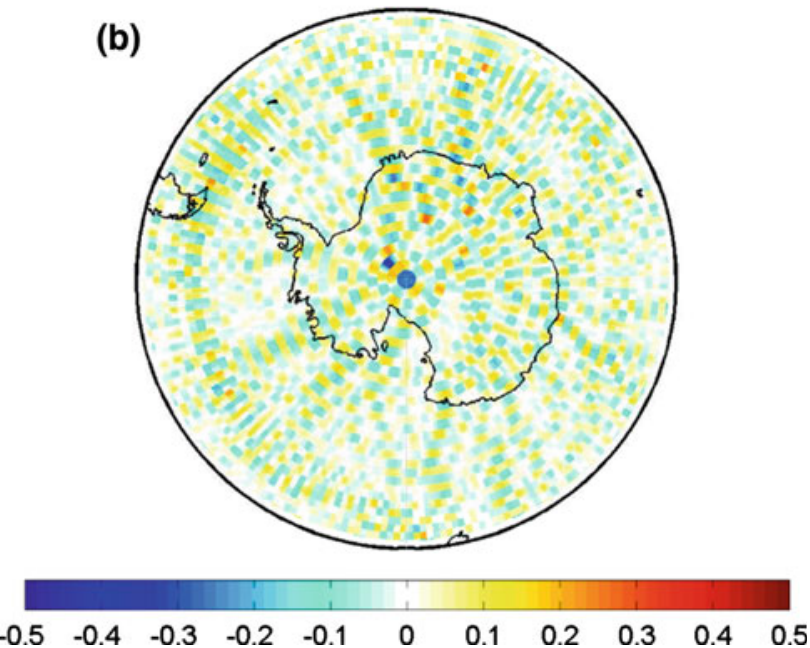

Fig. 10 a Static gravity field correction computed up to degree 120 from 11 months of actual noise (after band-pass filtering to highlight the range 9-23 $\mathrm{mHz}$ ) and $\mathbf{b}$ difference between GOCO02S and EIGENGL04C static gravity field models truncated at degree 120, in terms of geoid height $(\mathrm{m})$

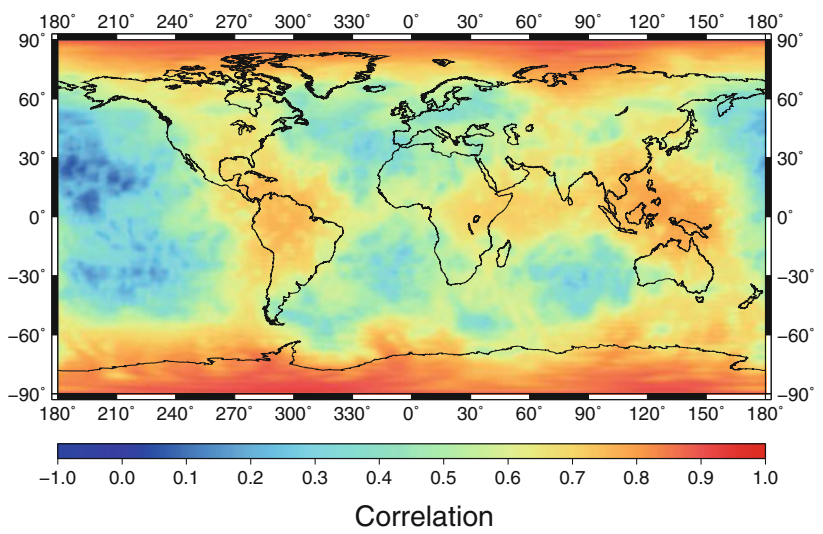

Fig. 11 Spatial correlation between: (i) computed correction to the static gravity field model EIGEN-GL04C and (ii) the difference between the state-of-the-art GOCO02 model and EIGEN-GL04C model (truncation at degree 120 is applied) 
tracks in the polar areas is large. This makes the sensitivity of the GRACE mission to gravity field signals more isotropic and, therefore, reduces the non-uniqueness of the gravity field recovery. An additional factor, which may also play a role, is a relatively high density of measurements in the polar areas. Thanks to the combination of these two factors, the computed correction to the static gravity field model EIGEN-GL04C leads to an actual improvement near the poles. As far as low-latitude areas are concerned, the intrinsic nonuniqueness of the GRACE mission, which is caused by its anisotropic sensitivity, leads to a situation where the computed correction is only one of many possible functions. It can explain the residual signal in the GRACE data, but cannot be treated as an actual improvement of the static model of the Earth's gravity field.

Actual noise corrected for the residual static field up to degree 180 is called thereafter "corrected actual noise". Only this type of actual noise is considered in the analysis in the further subsections.

\subsection{Contribution of ranging sensor errors}

The relatively strong noise at high frequencies, which is observed even after the subtraction of the residual static field contribution, is attributed to an imperfectness of the KBR sensor. In order to provide a further support of this statement, we generate synthetic realizations of ranging noise $\delta_{R}$. As input, we define noise $\mathrm{PSD}^{\frac{1}{2}}$ in terms of inter-satellite ranges. We assume that it is represented by a linear function in the logarithmic scale. On this basis, we generate realizations of random errors in ranges, transform them into range combinations with Eq. (16), and then introduce the appropriate data gaps. It is found that the best fit to the corrected actual noise $\mathrm{PSD}^{\frac{1}{2}}$ in the range $14-80 \mathrm{mHz}$ is obtained if $\mathrm{PSD}^{\frac{1}{2}}$ of the errors in ranges decreases with a rate of $-6.4 \mathrm{~dB}$ per decade (see Fig. 12a). Furthermore, we fine-tune the generated noise realizations by applying an individual scaling factor per month. As a result, the std of errors in ranges varies between 0.72 and $0.94 \mu \mathrm{m}$ (in average, $0.81 \mu \mathrm{m}$ ). The obtained stochastic properties of the errors in ranges are, in general, consistent with those known from literature (Kim and Tapley 2002; Frommknecht et al. 2006; Kim and Lee 2009).

The $\mathrm{PSD}^{\frac{1}{2}}$ of the corrected actual noise and of the synthetic ranging noise are presented for one of the months in Fig. 12b. As one can see, these two $\mathrm{PSD}^{\frac{1}{2}}$ are very close to each other above the frequency of $\sim 14 \mathrm{mHz}$. The other months also show a good consistency between the synthetic and corrected actual noise in this frequency range (not shown). The peak at the frequency of $30 \mathrm{mHz}$ observed in the corrected actual noise $\mathrm{PSD}^{\frac{1}{2}}$ is due to residual static field signal, as explained in the previous subsection. At the frequencies below $\sim 14 \mathrm{mHz}$, an increasing discrepancy between the
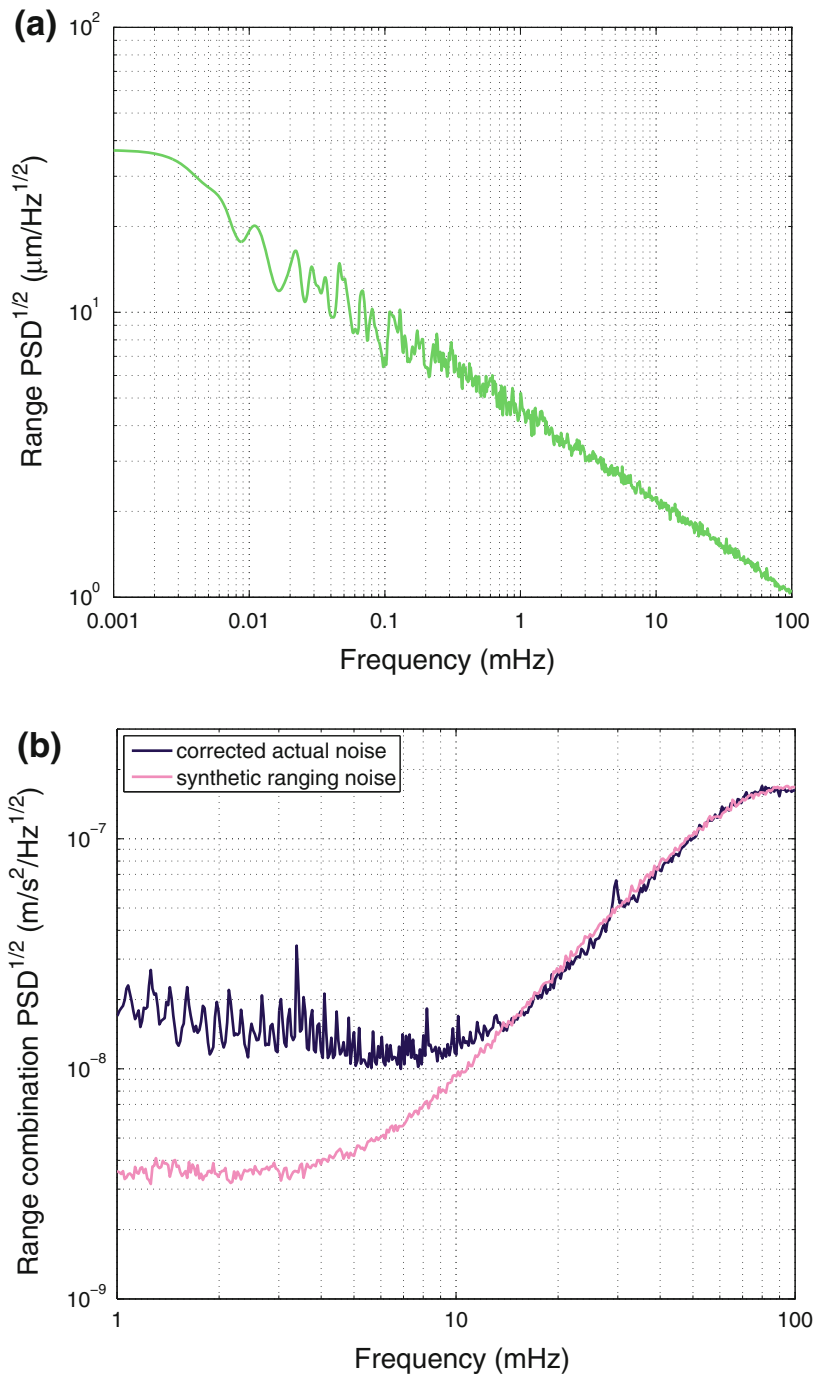

Fig. 12 a $\mathrm{PSD}^{\frac{1}{2}}$ of synthetic errors in ranges; $\mathbf{b} \mathrm{PSD}^{\frac{1}{2}}$ of the synthetic ranging noise obtained by the propagation of errors in ranges (in pink) and $\operatorname{PSD}^{\frac{1}{2}}$ of the corrected actual noise (in dark blue). The month under consideration is February, 2006

synthetic noise and the corrected actual one is observed. This suggests that some other contributors to the noise budget start to play a role there.

A comparison of synthetic and corrected actual noise is also performed in terms of gravity field parameters. In doing so, we apply a high-pass filter to the noise time series of both types, so that the contribution of frequencies below $23 \mathrm{mHz}$ is suppressed. After that, the noise realizations of both types are inverted into gravity field parameters as described above. It is worth mentioning that the frequency-dependent data weighting in this particular case is switched off to prevent downweighting of the high frequencies, which are the major focus of the conducted comparison. The computed gravity field parameters are represented in terms of equivalent water layer thickness (Wahr et al. 1998). It turns out that the spatial patterns of noise of the two considered types are sufficiently 

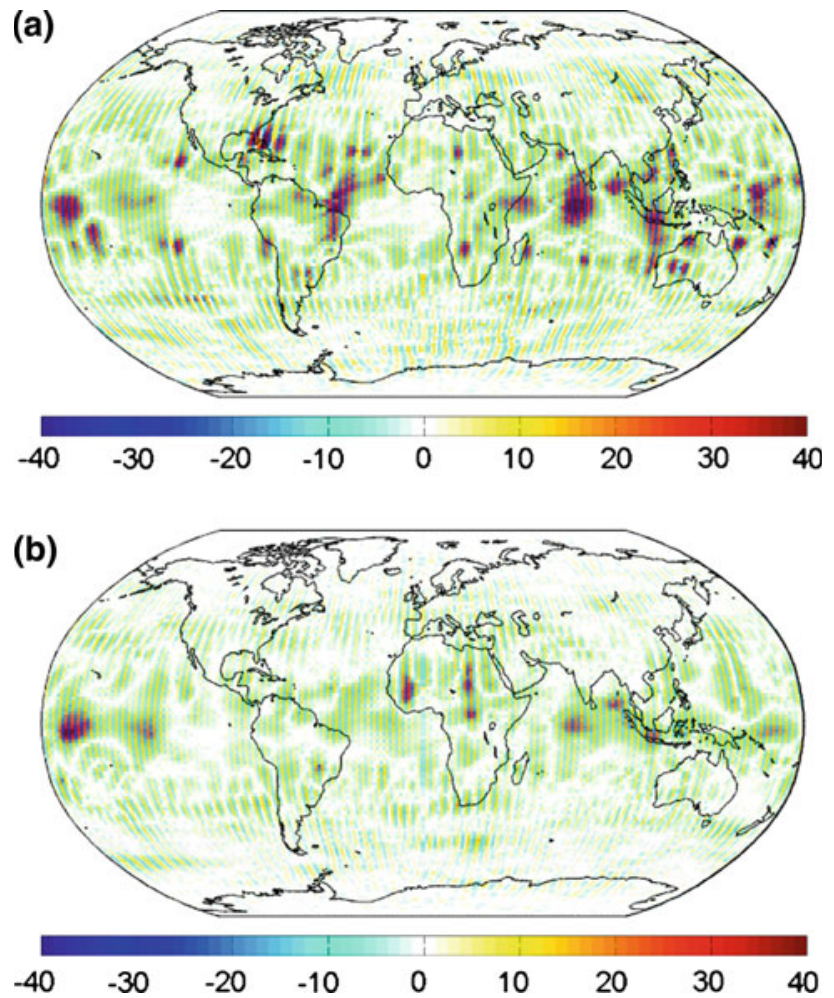

Fig. 13 High-pass filtered noise propagated into gravity field parameters and represented in terms of equivalent water layer thickness (m): a corrected actual noise (RMS $=8.1 \mathrm{~m}$ ) and $\mathbf{b}$ synthetic ranging noise $(\mathrm{RMS}=5.2 \mathrm{~m})$. The month under consideration is February, 2006

similar to each other (see, e.g., Fig. 13). In both cases, the noise forms along-track stripes, which vanish near the poles and reach maximum near the equator. Otherwise, the regions with relatively low and high noise are chaotically distributed over the globe. It is necessary to add that the total RMS error averaged over 11 months is somewhat larger in case of the corrected actual noise than in case of the synthetic one: 9.1 versus $5.2 \mathrm{~m}$, respectively. This discrepancy can be attributed to the fact that corrected actual noise still contains a residual gravity field signal, as indicated by the peak at degree 180. This signal is hardly visible in the frequency domain, but apparently still plays a large role in the spatial domain because it does not average out as the length of the time series increases.

\subsection{Contribution of inaccuracies in models of temporal gravity field variations}

In the present section, we analyze to which extent actual noise can be explained by the presence of errors in the exploited background models of temporal gravity field variations. We consider two potential sources of errors: (i) inaccuracies in the model of non-tidal mass transport in the atmosphere and ocean (AOD1B product); and (ii) an insufficiently accu- rate description of slow mass transport given by the DMT-1 model. Though the scope of the conducted analysis is very limited, it still allows some general conclusions to be drawn regarding the potential effect of errors in models of timevarying gravity field.

Errors in a model of non-tidal mass transport in the atmosphere and ocean are defined as $10 \%$ of residual signal, which is derived as the difference between the instantaneous signal and the monthly mean. In view of previous researches of errors in mass transport models, such an assumption can be considered as sufficiently reasonable (see, e.g., Thompson et al. 2004). Some authors applied in the past also more advanced approaches to quantify errors in models of nontidal mass transport in the atmosphere and ocean. One of the possible approaches is to consider the difference between two alternative meteorological models describing atmospheric pressure variations, which are the major contributor to mass transport of the considered type (Velicogna et al. 2001; Han 2004; Thompson et al. 2004). Another possible approach is to make use of the error estimations provided by a meteorological model itself (Zenner et al. 2010). For the purpose of our analysis, however, the approach we adopted is believed to be sufficiently adequate.

Of course, non-tidal mass re-distribution in the atmosphere and ocean is only one of mass transport processes; an inaccurate description of other processes contributes to the noise budget as well. Therefore, we find it important to consider also the DMT-1 model as a potential source of inaccuracies. This model is based on GRACE data themselves and, therefore, describes the total mass transport independently of its origin. An obvious limitation of the DMT-1 model stems from its limited temporal resolution. Like most of other GRACE-based models, it consists of monthly solutions, so that a mass variation within each particular month is assumed to be constant. The difference between the actual mass variation at a certain moment and the mean monthly value is a source of additional errors. In order to estimate its potential effect, we use an alternative scheme to derive mass variations in the time domain from the DMT-1 model. Instead of assuming that they are piece-wise constant, we use a quadratic spline approximation designed in such a way that the monthly mean computed on the basis of the spline is equal to the original value from the DMT-1 model. The difference between the two representations in the time domain is taken as the residual signal and used in our analysis.

The residual mass transport signals of both types (associated with the AOD1B product and with the DMT-1 model) are propagated into range combinations, as discussed in Sect. 2.2.6. The result is interpreted as synthetic noise of $\bar{\delta}_{\mathrm{GV}}$ type (noise caused by errors in time-varying gravity field models). The $\mathrm{PSD}^{\frac{1}{2}}$ computed on the basis of the obtained noise time series for February 2006 are shown in Fig. 14 (the results for other months look similar). 


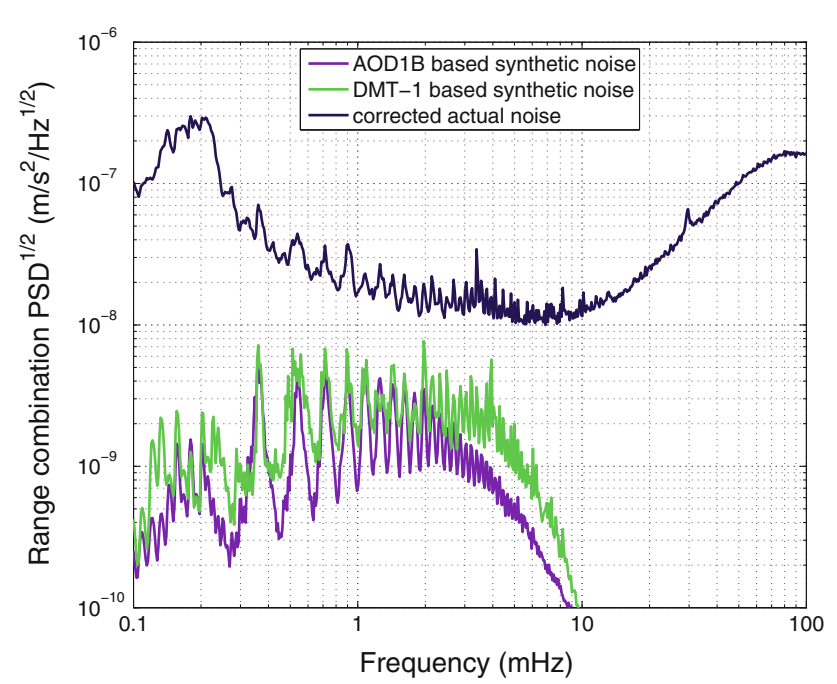

Fig. $14 \operatorname{PSD}^{\frac{1}{2}}$ of synthetic noise caused by potential errors in timevarying gravity field models: based on the AOD1B product (purple line), and based on the DMT-1 model (green line). PSD $\frac{1}{2}$ of corrected actual noise (dark blue line) is shown as a reference. The month under consideration is February, 2006

From a comparison of the presented $\mathrm{PSD}^{\frac{1}{2}}$ curves, one can conclude that synthetic noise of both types is at least one order of magnitude smaller than corrected actual noise. Errors in time-varying gravity field models may contribute to the noise budget substantially only if their level is about 10 times larger. In the AOD1B case, this means that the errors must be comparable with the signal itself, which seems to be an overly pessimistic assumption. Furthermore, even in that case the contribution of the errors under consideration must be limited to the frequency band from 1 to $3 \mathrm{mHz}$. At higher frequencies, $\mathrm{PSD}^{\frac{1}{2}}$ of synthetic noise of both types show a very rapid reduction, which can be explained by the upward continuation effect.

One may argue that noise associated with errors in models of temporal gravity field variations may not be stationary, so that an attempt to quantify it in terms of $\operatorname{PSD}^{\frac{1}{2}}$ might be misleading. In order to make our analysis more comprehensive, we propagate the synthesized noise realizations, as well as corrected actual noise, into gravity field parameters. To isolate the frequency range where synthetic noise is especially significant, the band-pass filtering (band $1-3 \mathrm{mHz}$ ) is consistently applied to the time series of synthetic and corrected actual noise. The results obtained in this way for February 2006 are shown in terms of equivalent water layer thickness in Fig. 15. One can see that in the spatial domain corrected actual noise also shows a different behavior than synthetic noise. Corrected actual noise reaches maximum near the equator, the RMS value averaged over 11 months being equal to $42.7 \mathrm{~m}$. On the contrary, synthetic noise based on the AOD1B product is maximal at the intermediate lati-
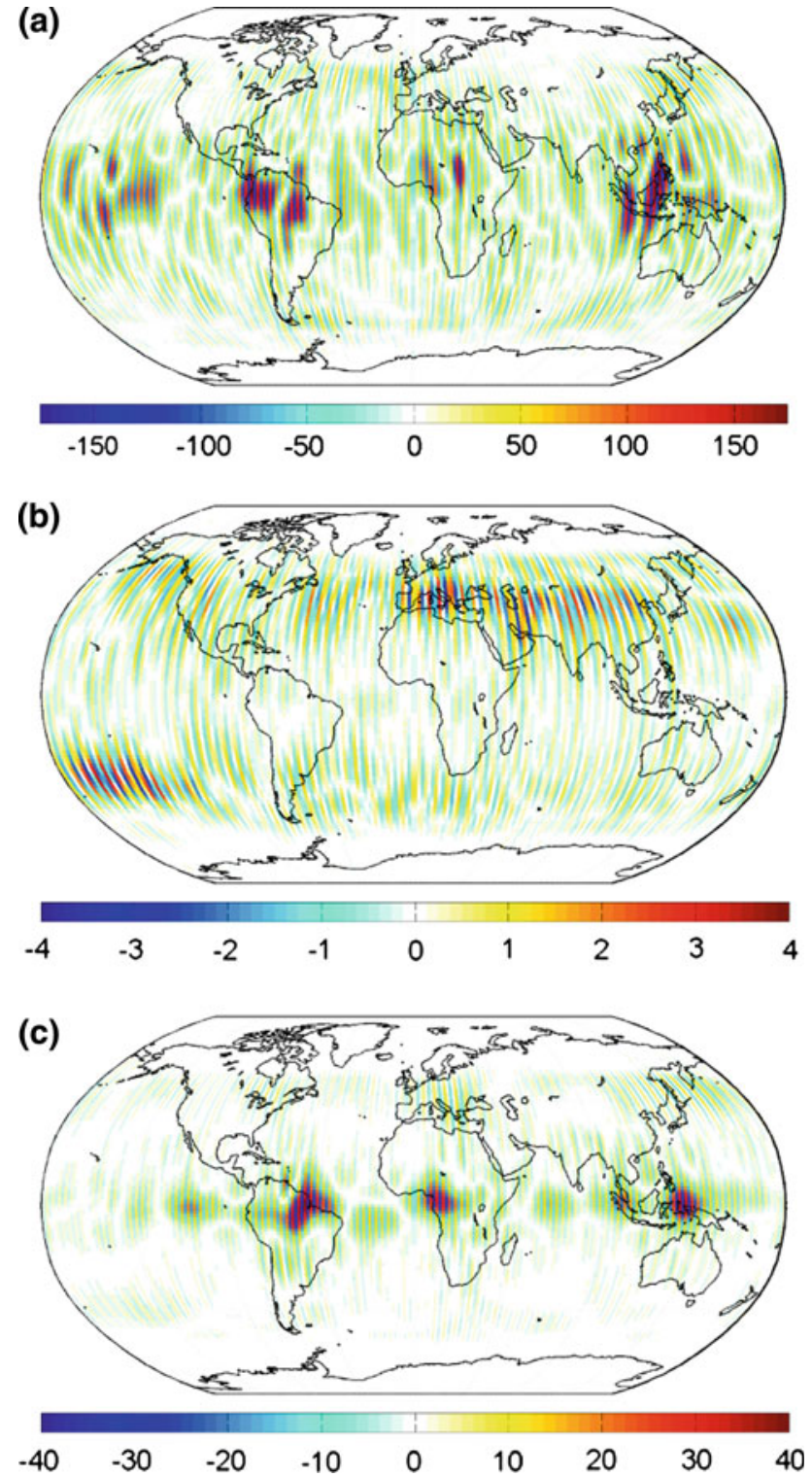

Fig. 15 Corrected actual noise and synthetic noise due to errors in models of temporal gravity field variations, propagated into gravity field parameters after a band-pass filtering (band $1-3 \mathrm{mHz}$ ) and represented in terms of equivalent water layer thickness $(\mathrm{m})$ : a case of corrected actual noise (RMS $=31.6 \mathrm{~m})$; $\mathbf{b}$ case of synthetic noise based on the AOD1B product $(\mathrm{RMS}=0.6 \mathrm{~m})$; and $\mathbf{c}$ case of synthetic noise based on the DMT-1 model (RMS $=4.8 \mathrm{~m}$ ). The month under consideration is February, 2006

tudes and is much smaller at the equator. Furthermore, the RMS value of noise of this type (average over 11 months) is only $0.5 \mathrm{~m}$. One may argue that in reality noise in the AOD1B product may not be proportional to signal and, therefore, may result in a very different spatial pattern. Nevertheless, to explain the level of corrected actual noise near the equator, we need to assume that noise in AOD1B product in the equatorial area exceeds signal by orders of magnitude, which is very unlikely. As far as synthetic noise based on the DMT- 
1 model is concerned, the resulting spatial pattern is much closer to that in case of corrected actual noise. Nevertheless, the level of synthetic noise is still too low. The RMS value of this noise averaged over 11 months is equal to $7.0 \mathrm{~m}$, which is an order of magnitude smaller than in the case of corrected actual noise.

The conducted comparison allows us to conclude that inaccuracies in the AOD1B product as well as a limited temporal resolution of the DMT-1 model are, most probably, not the dominant sources of noise in the GRACE data, including the frequency range from 1 to $3 \mathrm{mHz}$. Furthermore, it is unlikely that errors in the other background mass transport models significantly exceed the residual signals considered in our analysis. Thus, no evidence is found that GRACE-based solutions significantly suffer from errors in background time-varying gravity models used for temporal de-aliasing. This does not exclude the option, however, that such errors still play a role locally, i.e., in limited regions where their level significantly exceeds the average one.

We have also propagated the realizations of synthetic noise $\bar{\delta}_{\mathrm{GV}}$ into gravity field parameters without a band-pass filtering to see the total effect this noise may have. The obtained results (not shown) turn out to be very similar to those obtained with the band-pass filtering, both in terms of the RMS and the spatial pattern.

\subsection{Contribution of random noise of unknown origin}

After the completion of the analysis presented in the previous sections, we have to admit that the origin of actual noise in the frequency range $1-9 \mathrm{mHz}$ remains unclear. However, we can, at least, investigate whether the impact of actual noise in this range is similar to that of random stationary noise characterized by the same $\operatorname{PSD}^{\frac{1}{2}}$. To that end, we generate realizations of empirically defined stationary random noise: the $\mathrm{PSD}^{\frac{1}{2}}$ of this noise are close to those of corrected actual noise. A band-pass filtering (band $1-9 \mathrm{mHz}$ ) is consistently applied to the realizations of synthetic and corrected actual noise to suppress the contribution of irrelevant frequency bands. A comparison of $\mathrm{PSD}^{\frac{1}{2}}$ of the computed noise realizations in the target band has demonstrated that they may differ by as much as $20 \%$, which can be explained as the effect of filtering and the gaps introduced into the synthetic noise. Therefore, we scale the synthetic noise in order to make its $\mathrm{PSD}^{\frac{1}{2}}$ close to that of the corrected actual noise in the target frequency band. Finally, realizations of both synthetic and corrected actual noise are propagated into gravity field parameters. The results obtained for February 2006 are shown (in terms of equivalent water layer thickness) in Fig. 16.

One can see that the spatial patterns of the propagated noise as well as the RMS values are very similar for the actual and the synthetic noise realizations in February 2006.
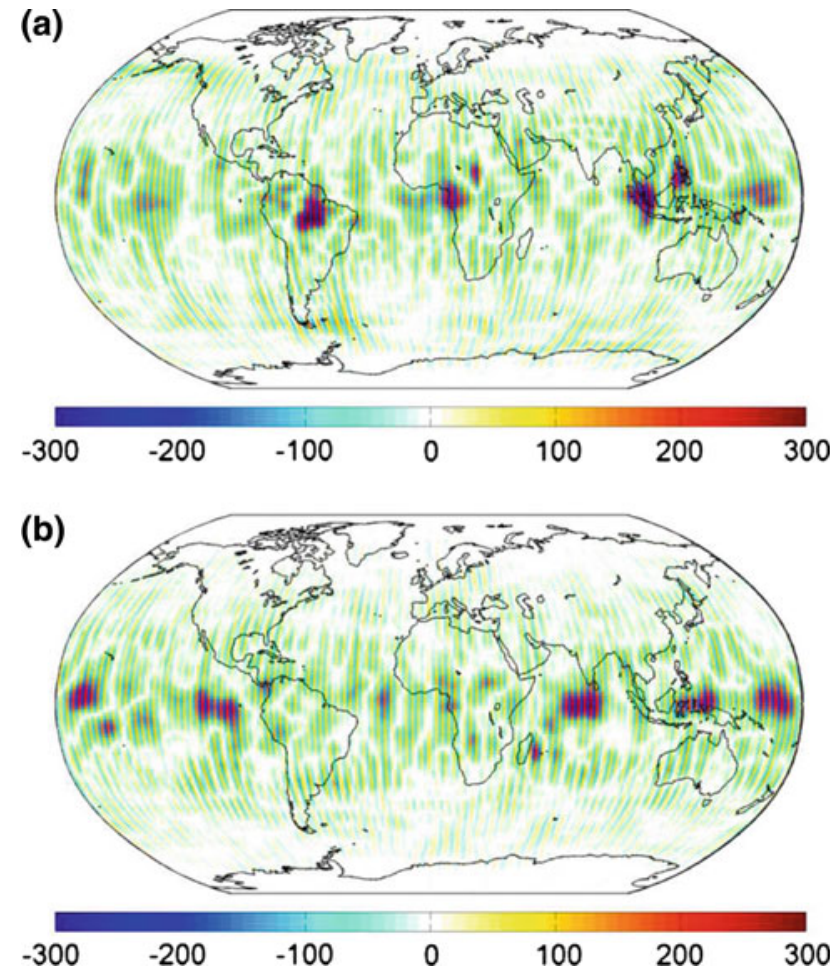

Fig. 16 Corrected actual noise and empirically defined synthetic random noise propagated into gravity field parameters after a band-pass filtering (band 1-9 mHz), in terms of equivalent water layer thickness $(\mathrm{m})$ : $\mathbf{a}$ case of corrected actual noise (RMS $=51.8 \mathrm{~m})$; $\mathbf{b}$ case of synthetic random noise $(\mathrm{RMS}=53.3 \mathrm{~m})$. The month under consideration is February, 2006

The same result was obtained also for other months (the RMS values averaged over 11 months are equal 61.4 and $59.1 \mathrm{~m}$ for the corrected actual noise and synthetic random noise, respectively). Thus, the assumption that actual noise in the frequency band $1-9 \mathrm{mHz}$ is stationary cannot be proven false. This is an additional evidence that the primary origin of this noise is not related to errors in the background models describing the Earth's static or time-varying gravity field.

\subsection{Integrated effect of synthetic noise of different origin}

To conclude our analysis, we estimate the integrated effect of synthetic noise of four different types considered in Sect. 4 . This concerns

1. Ranging noise $\sigma_{R}$;

2. Noise $\sigma_{\mathrm{GV}}$ caused by errors in the AOD1B product;

3. Noise $\sigma_{\mathrm{GV}}$ caused by errors in the DMT- 1 model;

4. Empirically defined stationary random noise occupying the frequency band $1-9 \mathrm{mHz}$.

$\mathrm{PSD}^{\frac{1}{2}}$ of noise of the considered types, as well as of the corrected actual noise, are shown in Fig. 17a.

The considered realizations of synthetic noise are propagated into gravity field parameters. It is important to point 

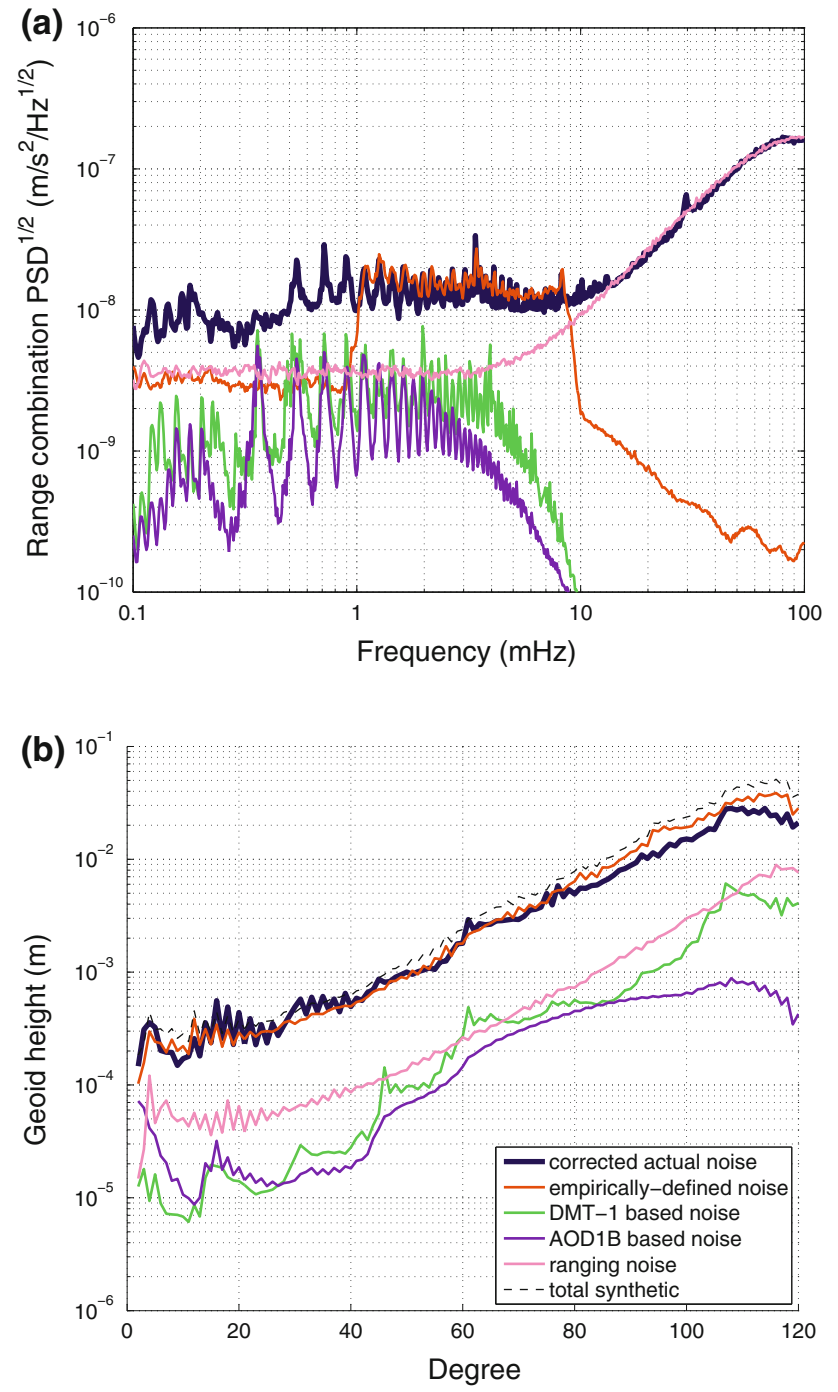

Fig. 17 a PSD $^{\frac{1}{2}}$ of the considered realizations of synthetic noise associated with middle- and high-frequencies (February 2006) and b results of propagating the considered realizations into gravity field parameters and shown in terms of RMS geoid heights per degree (average over all 11 months). The considered types of synthetic noise are: ranging noise $\sigma_{R}$ (in pink); noise $\sigma_{\mathrm{GV}}$ caused by errors in the AOD1B product (in purple); noise $\sigma_{\mathrm{GV}}$ caused by errors in the DMT-1 model (in green); empirically defined stationary random noise occupying the frequency band $1-9 \mathrm{mHz}$ (in brown); sum of all four synthetic noise types (dashed grey line in the bottom plot). Results based on the corrected actual noise are shown as well (dark blue lines)

out that band-pass filtering is not applied in the context of this analysis (except for the case of empirically defined random noise, which is the result of a band-pass filtering by definition). For a comparison, corrected actual noise is also propagated into gravity field parameters, the low-frequency contents being suppressed with the procedure explained at the beginning of Sect. 4, cf. Eq. (52). The obtained results are presented in terms of RMS geoid height per degree (averaged over 11 months) in Fig. 17b. The effects of synthetic noise of various types are shown in Fig. 17b both separately and after the summation. From this figure, it is obvious that empirically defined random noise, which mimics actually observed noise of unknown origin in the frequency band from 1 to $9 \mathrm{mHz}$, is the dominant contributor to the errors in GRACE-based gravity field models. The contribution of middle- and high-frequency noise of other types (due to ranging errors and errors in background models of time-varying gravity fields) is an order of magnitude lower. Such a high impact of empirically defined random noise can be explained by the fact that it spans the frequency band from 5.4 to 49 cycles per revolution, i.e., overlaps with that containing the major part of the gravity field spectrum. It is also remarkable that this noise remains dominant up to the maximum considered degree (120). Most probably, this is because this noise manifests itself in the form of along-track stripes in the spatial domain (see Fig. 16b). Thus, even high-degree coefficients are severely distorted if they are sectorial and nearly-sectorial. It is worth adding that the observed impact of ranging noise alone is close to the baseline errors (Kim 2000), which reflect the pre-launch expectations regarding the GRACE mission performance.

\section{Discussion and conclusions}

The analysis presented in this paper leads to a better understanding of noise in the KBR data acquired by the GRACE satellite mission. It has been demonstrated that noise at lowfrequencies can be mostly explained by inaccuracies in the computed orbits of GRACE satellites, which exist even if state-of-the-art models of forces acting on the satellites are used. First, errors in the orbits lead to an inaccurate estimation of the radial component of the inter-satellite velocities. This results in errors in centrifugal accelerations, which have to be taken into account when the measured range-accelerations are linked with inter-satellite accelerations. This is the most important contributor to the error budget among those related to inaccuracies in satellite orbits. The second contributor, which has only a slightly smaller effect, is caused by inaccurately known inter-satellite distances when they are derived from computed satellite orbits. The presence of errors in the estimated distances may lead to an inaccurate computation of the forces acting on the satellites. Particularly, the contribution of the zero-degree term of the Earth's gravitational field to the force model is miscalculated. The third contributor, which is about one order of magnitude smaller according to the conducted analytic analysis, is caused by inaccurately known radial positions of the GRACE satellites.

The other sources of noise play a role only at the intermediate and high frequencies. Unfortunately, the dominant source of noise in the range of intermediate frequencies $(1-9 \mathrm{mHz})$ is not fully understood yet. In spite of that, the conducted analysis has led to a number of important findings. 
We have found that the analyzed realizations of data noise contained a residual static field signal. We have shown that the inversion of produced noise realizations into a static field update allows this signal to be reduced. On the other hand, it is important to realize that the implementation of this update not necessarily improves the static field model. In the considered example, the update is somewhat meaningful in the polar areas, but is less successful at low latitudes. Most probably, this can be explained by the intrinsic non-uniqueness of the GRACE mission caused by its anisotropic sensitivity: different updates can explain the residual signal in the GRACE data equally well. In other words, the situation is somewhat similar to that already existed in pre-GRACE era, when the available satellite data allowed "tailored" gravity field models to be produced that perfectly explained those data. However, those tailored models could be rather different from the real field, which became clear as soon as new data became available.

Furthermore, we have demonstrated that the contribution of ranging sensor errors becomes dominant only at rather high frequencies (not less than $14 \mathrm{mHz}$ ). After a propagation of resulting high-frequency noise in gravity field parameters, the observed effect is about one order of magnitude lower than that of actual noise. We have also shown that errors in models of time-varying gravity field cannot play a significant role in the observed noise spectrum. Furthermore, we have demonstrated that those errors cannot, in general, explain noise in gravity field models obtained by inversion of GRACE data.

Now, let us discuss whether the conclusions drawn on the basis of the conducted study can be considered as sufficiently general. As explained in Sect. 2.2.4, the range combinations we introduce can be considered as a finite-difference approximation of inter-satellite accelerations. On the one hand, those data can be directly related to the forces acting on the satellites (including the Earth's gravitational field). On the other hand, they can be derived from double-differentiated intersatellite ranges corrected for the contribution of centrifugal accelerations, cf. Eq. (9). Let us assume for a moment that the latter correction can be made in an error-free way. In this case, the exploited functional model would provide a unique link between the unknown parameters and the double-differentiated observations (namely, inter-satellite ranges). As shown by Ditmar and van Eck van der Sluijs (2004), the solution based on this functional model coincides with the solution obtained by the inversion of the original observations (i.e., ranges) themselves or their first-order derivatives (i.e., rangerates), provided that the statistically optimal estimation procedure is followed. In reality, the correction for centrifugal accelerations is not error-free. However, the corresponding noise manifests itself at low degrees (below $1 \mathrm{mHz}$ ) only. Therefore, all the results we obtained for the intermediate and high frequencies (the focus of Sect. 4) should be considered as applicable to optimal estimation procedures in general, no matter whether ranges, range-rates, or range-accelerations are used as input. Of course, the conducted study does not allow anything to be said about non-optimal procedures (e.g., lacking a proper data weighting). For instance, a non-optimal procedure might show a much stronger influence of the ranging sensor noise than was observed in the current study. However, all such problems of non-optimal procedures must be considered as procedure-specific ones and should be treated on a case-by-case basis.

As far as errors in the estimated orbits are concerned, the exploited functional model cannot be considered as sufficiently representative in general, because it handles orbits as pre-defined objects. In most of other functional models, orbits of GRACE satellites are considered as functions of unknown parameters. Of course, those functional models may lead to different values of gravity field parameters than the exploited one. Thus, our attempts to propagate orbit noise into gravity field parameters would not produce results of a general interest, and for this reason we did not make such attempts. Nevertheless, the presence of relatively strong low-frequency noise due to inaccuracies in the computed orbits (particularly, centrifugal acceleration noise) allows us to suggest that this noise may play a significant role in a general case as well. This can be further understood from the fact that the GRACE observation principle is nothing but gravity gradiometry. Thanks to a 200-km separation between the sensors, the GRACE gradiometer has an extremely high sensitivity (in fact, such a large separation allows GRACE data to be treated as gravity gradients only in the context of lowest spatial frequencies; otherwise, these data must be interpreted as gravitation differences). Another peculiar feature of the GRACE gradiometer is the presence of only one arm, so that at maximum three gravity gradient components $(x x, x y$, and $x z)$ out of nine can be determined. In addition, the sensor positions are not rigidly fixed with respect to each other, so that they need to be continuously tracked. The exploited ranging technique allows relative position changes to be determined only along the $\mathrm{x}$-axis. Thus, only the $x x$ gravity gradient component can be measured with a high accuracy. In spite of all the peculiarities mentioned above, the GRACE gradiometer, like any other gravity gradiometer, senses not only the gravity gradients, but also the inertial force, namely the centrifugal force caused by the rotation of the line connecting the sensors (i.e., the line-of-sight). And just like in case of any other gravity gradiometer, this force needs to be corrected for, for which purpose independent information about the frame rotation (i.e. about the radial component of the inter-satellite velocity vector) must be available. Inaccuracies in such a correction term definitely reduce the overall accuracy of the measurements collected by a gradiometer.

The conducted analysis allows certain ways to be identified towards increasing the accuracy of models derived from 
inter-satellite ranging data (to be) collected by GRACE and GRACE follow-on missions. In the context of GRACE, it is advisable to increase further the accuracy of the orbit determination (particularly, to determine more accurately the inter-satellite velocities). Possible ways to achieve this goal are (i) to improve further the accuracy of GPS data processing; (ii) to incorporate KBR data in the orbit determination process (if this is not done yet); (iii) to experiment with additional empirical parameters that may absorb the observed orbit inaccuracies; (iv) to optimize the duration of orbital arcs; and (v) to improve further the accuracy of background force models. In the context of GRACE follow-on missions, a special mission design can be considered, so that the influence of centrifugal accelerations onto the data is minimized. For instance, a cartwheel satellite formation (Wiese et al. 2009) is a very attractive option in this sense, because it allows temporal variations of the line-of-sight orientation in an inertial frame to be reduced to minimum.

Another point of attention is the definition of the static field in mass transport monitoring. Inaccuracies in the exploited static field model can manifest themselves as a residual signal in the input data. One may argue that this issue is not critical because the presence of such a signal simply causes a static bias in the time-varying gravity field solutions. It is, however, not impossible that this residual signal propagates into different solutions differently due to a changing ground-track pattern and the intrinsic non-uniqueness of gravity field recovery from GRACE data (particularly, at high degrees). Then, a fictitious time-varying signal may show up. A possible solution to this problem is to start mass transport recovery from computing a static gravity field model up to a very high spherical harmonic degree (in any case, above degree 180), so that all static field signal in the data is absorbed as completely as possible. It is important to stress that the static model produced in this way must be treated as a tailored GRACE-based model, which can be far from the reality. One may argue that compiling such a tailored static field model is not needed if a state-of-the-art model is exploited (e.g., the one produced by a combined inversion of GRACE and GOCE data). It is important to realize, however, that a similar problem may also be faced later, in the context of future GRACE follow-on missions. If such a mission is even more sensitive than GRACE and, at the same time, also suffers from a non-uniqueness, it can happen that residual static field signal is still present in the data, whereas the spherical harmonic degrees involved are too high to benefit from GOCE data.

The conducted analysis leads also to a more general recommendation: it is worth designing GRACE follow-on missions in such a way that the non-uniqueness of gravity field recovery is minimized. In particular, an anisotropic sensitivity of the future mission would be a highly undesirable feature, as it is one of the major sources of this non-uniqueness. This will reduce static field signals that cannot be uniquely explained by the models produced on the basis of the collected data. Another potential benefit of such a mission design is that it will presumably reduce the impact of data noise in general. Then, lower noise in gravity field models can be achieved compared with the GRACE-type design, even if noise in the Level-1B data is similar.

Another aspect tackled in the paper is the accuracy of the background models describing time-varying gravity field (e.g., the AOD1B product). One might interpret the fact that we could not observe a significant influence of those errors as a recommendation not to care about a further improvement of those models. We stress that such an interpretation would be erroneous. First of all, it is not impossible that those errors still play a role in limited geographical areas (revealing such an effect would require a more detailed analysis of errors in background force models, which is beyond the scope of the presented paper). Second, more accurate force model will be beneficial for precise orbit determination, so that low-frequency noise in the data might be reduced in this way. Finally, improvement of background force models is definitely needed in the context of GRACE follow-on missions as one of the necessary pre-requisites to improve the accuracy of mass transport models to be produced.

Finally, investigation of the remaining sources of noise in GRACE data must be continued (in particular those responsible for the noise observable in the frequency range 1-9 $\mathrm{mHz}$ ). For instance, recently revealed inaccuracies in satellite attitude data (Horwath et al. 2010) can be considered as a potential source of such noise. The effect of possible inaccuracies in the estimates of accelerometer calibration parameters is also worth investigating. We stress that complete understanding of the noise budget would be important not only for GRACE data processing, but also in designing GRACE follow-on missions.

Acknowledgments High-precision kinematic orbits of GRACE satellites used as an input in the presented study were computed by Dr. Qile Zhao. The script for a spline approximation of piece-wise constant monthly solutions in the time domain was written by Dr. Christian Siemes. We thank also the GNSS Research and Engineering Center of Wuhan University for providing us with the PANDA software for satellite orbit integration. In addition, we thank three anonymous reviewers and prof. Jürgen Kusche, the handling editor, for the help in improving the manuscript. The work was sponsored by the Stichting Nationale Computerfaciliteiten (National Computing Facilities Foundation, $\mathrm{NCF}$ ) for the use of supercomputer facilities, with financial support from the Nederlandse organisatie voor Wetenschappelijk Onderzoek (Netherlands Organization for Scientific Research, NWO).

Open Access This article is distributed under the terms of the Creative Commons Attribution Noncommercial License which permits any noncommercial use, distribution, and reproduction in any medium, provided the original author(s) and source are credited. 


\section{References}

Bettadpur SV (2007) UTCSR level-2 processing standards document for Level-2 product release 0004. Center for Space Research, University of Texas at Austin, Austin

Case K, Kruizinga G, Wu SC (2004) GRACE level 1B data product user handbook. JPL D-22027. GRACE 327-733. JPL

Ditmar P, van Eck van der Sluijs AA (2004) A technique for Earth's gravity field modeling on the basis of satellite accelerations. J Geod 78:12-33

Ditmar P, Kuznetsov V, van Eck van der Sluijs AA, Schrama E, Klees R (2006) 'DEOS_CHAMP-01C_70': a model of the Earth's gravity field computed from accelerations of the CHAMP satellite. J Geod 79:586-601

Flechtner F (2007) AOD1B product description document for product reslease 01 to 04. GeoForschungsZentrum Potsdam, Germany

Flechtner F, Dahle C, Neumayer KH, König R, Förste C (2010) The release 04 CHAMP and GRACE EIGEN gravity models. In: Flechtner F (ed) et al System Earth via geodetic-geophysical space techniques. Adv technologies in Earth sciences, part 1. Springer, Berlin pp 41-58. doi:10.1007/978-3-642-10228-8_4

Flury J, Bettadpur S, Tapley BD (2008) Precise accelerometry onboard the grace gravity field satellite mission. Adv Space Res 42:1414 1423

Förste C, Schmidt R, Stubenvoll R, Flechtner F, Meyer U, König R, Neumayer H, Biancale R, Lemoine JM, Bruinsma S, Loyer S, Barthelmes F, Esselborn S (2008) The GeoForschungsZentrum Potsdam/Groupe de Recherche de Geodesie Spatiale satellite-only and combined gravity field models: EIGEN-GL04S1 and EIGENGL04C. J Geod 82:331-346

Frommknecht B, Fackler U, Flury J (2006) Integrated sensor analysis GRACE. In: Fluty J, Rummel R, Reigber C, Rotacher M, Boedeker G, Schreiber U (eds) Observation of the Earth system from space. Springer, Berlin pp 99-113

Goiginger H, Hoeck E, Rieser D, Mayer-Guerr T, Maier A, Krauss S, Pail R, Fecher T, Gruber T, Brockmann JM, Krasbutter I, Schuh WD, Jaeggi A, Prange L, Hausleitner W, Baur O, Kusche J (2011) The combined satellite-only global gravity field model GOCO02S. Presented at the 2011 General Assembly of the European Geosciences Union, Vienna, Austria, April 4-8, 2011

Han SC (2004) Efficient determination of global gravity field from satellite-to-satellite tracking mission. Celest Mech Dyn Astron 88:69-102

Han SC, Jekeli C, Shum CK (2004) Time-variable aliasing effects of ocean tides, atmosphere, and continental water mass on monthly mean grace gravity field. J Geophys Res 109:b04403. doi:10.1029/ 2003JB002501

Horwath M, Lemoine JM, Biancale R, Bourgogne S (2010) Improved GRACE science results after adjustment of geometric biases in the Level-1B K-band ranging data. J Geod. doi:10.1007/s00190-0100414-2

Ilk KH, Feuchtinger M, Mayer-Gürr T (2005) Gravity field recovery and validation by analysis of short arcs of a satellite-to-satellite tracking experiment as CHAMP and GRACE. In: Sansò F (ed) A window on the future of geodesy. IUGG General Assembly 2003, June 30-July 11 2003, Sapporo, Japan. International Association of Geodesy Symposia, vol 128. Springer, Berlin, Heidelberg, New York, pp 189-194

Kim J (2000) Simulation study of a low-low satellite-to-satellite tracking mission. $\mathrm{PhD}$ thesis, The University of Texas at Austin

Kim J, Lee SW (2009) Flight performance analysis of grace k-band ranging instrument with simulation data. Acta Astronautica 65(11-12):1571-1581. doi:10.1016/j.actaastro.2009.04.010. http://www.sciencedirect.com/science/article/B6V1N4WCSYT8-2/2/957dbf6f9322aeb06b9e8469113791d9
Kim J, Tapley BD (2002) Error analysis of a low-low satellite-tosatellite tracking mission. J Guidance Control Dyn 25(6):07315090. doi: $10.2514 / 2.4989$

Klees R, Ditmar P (2004) How to handle colored noise in large leastsquares problems in the presence of data gaps?. In: Sansò F (ed) V Hotine-Marussi symposium on mathematical geodesy. International Association of Geodesy Symposia, vol 127. Springer, Berlin, Heidelberg, New York pp 39-48

Klees R, Ditmar P, Broersen P (2003) How to handle colored observation noise in large-scale least-squares problems. J Geod 76:629640

Klees R, Rervtova EA, Gunter B, Ditmar P, Oudman E, Winsemius HC, Savanije HH (2008) The design of an optimal filter for monthly grace gravity field models. Geophys J Int 175:417-432

Lemoine JM, Bruinsma S, Loyer S, Biancale R, Marty JC, Perosanz F, Balmino G (2007) Temporal gravity field models inferred from GRACE data. Adv Space Res 39:1620-1629

Liu X (2008) Global gravity field recovery from satellite-to-satellite tracking data with the acceleration approach. PhD thesis, Netherlands Geodetic Commission, Publications on Geodesy, 68, Delft, The Netherlands

Liu X, Ditmar P, Siemes C, Slobbe DC, Revtova E, Klees R, Riva R, Zhao Q (2010) DEOS mass transport model (DMT-1) based on grace satellite data: methodology and validation. Geophys J Int 181(2):769-788

Luthcke SB, Rowlands DD, Lemoine FG, Klosko SM, Chinn D, McCarthy JJ (2006) Monthly spherical harmonic gravity field solutions determined from GRACE inter-satellite range-rate data alone. Geophys Res Lett 33:102402. doi:10.1029/2005GL024846

Lyard F, Lefevre F, Letellier T, Francis O (2006) Modelling the global ocean tides: Insights from FES2004. Ocean Dyn 56:394-415

McCarthy DD, Petit G (2004) IERS conventions (2003). IERS technical note, 32. Verlag des Bundesamts für Kartographie und Geodäsie, Frankfurt am Main

Pavlis NK, Holmes SA, Kenyon SC, Factor JK (2008) An Earth gravitational model to degree 2160: EGM2008. Oral presentation at EGU General Assembly 2008, Vienna, Austria, April 13-18, 2008

Pierce R, Leitch J, Stephens M, bender P, Nerem R (2008) Intersatellite range monitoring using optical interferometry. Appl Opt 47(27):5007-5018

Ray RD, Luthcke SB (2006) Tide model errors and grace gravimetry: towards a more realistic assessment. Geophys J Int 167: $1055-1059$

Rummel R (1979) Determination of short-wavelength components of the gravity field from satellite-to-satellite tracking or satellite gradiometry: an attempt to an identification of problem areas. Man Geodyn 4(2):107-148

Sneeuw N, Sharifi MA, Keller W (2008) Gravity recovery from formation flight missions. In: Xu P, Liu J, Dermanis A (eds) V Hotine-Marussi symposium on mathematical geodesy. International Association of Geodesy Symposia, vol 132. Springer, Berlin pp 29-34

Tapley BD, Bettadpur S, Ries JC, Thompson PF, Watkins MM (2004) GRACE measurements of mass variability in the Earth system. Science 294:2342-2345

Tapley B, Ries J, Bettadpur S, Chambers D, Cheng M, Condi F, Gunter B, Kang Z, Nagel P, Pastor R, Poole S, Wang F (2005) GGM02an improved Earth gravity field model from GRACE. J Geod 79:467-478

Thompson PF, Bettadpur SV, Tapley BD (2004) Impact of short period, non-tidal, temporal mass variability on GRACE gravity estimates. Geophys Res Lett 31:106619. doi:10.1029/2003GL019285

van Dam T, Visser P, Sneeuw N et al (2008) Monitoring and modeling individual sources of mass distribution and transport in the Earth system by means of satellites. Final report, ESA Contract 20403 
Velicogna I, Wahr J, den Dool HV (2001) Can surface pressure be used to remove atmospheric contributions from GRACE data with sufficient accuracy to recover hydrological signals? J Geophys Res 106(B8):16,415-16,434

Wahr J, Molenaar M, Bryan F (1998) Time variability of the Earth's gravity field: hydrological and oceanic effects and their possible detection using GRACE. J Geophys Res 103(B12):30,205-30,229

Wiese DN, Folkner WM, Nerem RS (2009) Alternative mission architectures for a gravity recovery satellite mission. J Geod 83:569-581
Zenner L, Gruber T, Jäggi A, Beutler G (2010) Propagation of atmospheric model errors to gravity potential harmonics: impact on GRACE de-aliasing. Geophys J Int 182:797-807

Zhao Q (2004) Research on precise orbit determination theory and software for both GPS navigation constellation and LEO satellites. $\mathrm{PhD}$ thesis, School of Geodesy and Geomatics, Wuhan University, Wuhan, China (in Chinese with an abstract in English) 